\title{
QUEEN'S
UNIVERSITY
BELFAST
}

\section{Underlay Cognitive Multihop MIMO Networks With and Without Receive Interference Cancellation}

Al-Qahtani, F. S., Radaydeh, R. M., Hessien, S., Duong, T. Q., \& Alnuweiri, H. (2016). Underlay Cognitive Multihop MIMO Networks With and Without Receive Interference Cancellation. IEEE Transactions on Communications. https://doi.org/10.1109/TCOMM.2016.2627574

Published in:

IEEE Transactions on Communications

Document Version:

Peer reviewed version

Queen's University Belfast - Research Portal:

Link to publication record in Queen's University Belfast Research Portal

Publisher rights

(c) 2016 IEEE. Personal use of this material is permitted. Permission from IEEE must be obtained for all other uses, in any current or future media, including reprinting/republishing this material for advertising or promotional purposes, creating new collective works, for resale or redistribution to servers or lists, or reuse of any copyrighted component of this work in other works

\section{General rights}

Copyright for the publications made accessible via the Queen's University Belfast Research Portal is retained by the author(s) and / or other copyright owners and it is a condition of accessing these publications that users recognise and abide by the legal requirements associated with these rights.

Take down policy

The Research Portal is Queen's institutional repository that provides access to Queen's research output. Every effort has been made to ensure that content in the Research Portal does not infringe any person's rights, or applicable UK laws. If you discover content in the Research Portal that you believe breaches copyright or violates any law, please contact openaccess@qub.ac.uk. 


\title{
Underlay Cognitive Multihop MIMO Networks With and Without Receive Interference Cancellation
}

\author{
Fawaz S. Al-Qahtani, Member, IEEE, Redha M. Radaydeh, Senior Member, IEEE, Salah Hessien, \\ Trung Q. Duong, Senior Member, IEEE, and Hussein Alnuweiri, Senior Member, IEEE,
}

\begin{abstract}
This paper investigates the impact of primary network interference on the performance of cognitive multihop secondary network under various multiple-input multiple-output MIMO approaches per hop. Specifically, the cognitive system involves a secondary network with MIMO relays that use the amplify-and-forward (AF) protocol, and each of which shares the same spectrum resources of multiple primary users (PUs) transmit and receive stations. Two different receive array conditions, and hence processing approaches, per hop in the secondary network are treated separately, which are maximal ratio combining (MRC) for sufficiently spaced receive antennas to provide receive diversity gain and interference cancellation (IC) for insufficiently spaced antennas to reduce the effect of PUs interference. The latter approach involves two different algorithms that vary in terms of complexity and achieved performance, which are dominant receive interference cancellation (DRIC) and adaptive receive interference cancellation (ARIC). Moreover, for both approaches, the transmit array gain is achieved per hop through the low-complexity transmit antenna selection (TAS). In doing so, new analytical results for multihop secondary network's end-to-end outage probability are developed. Moreover, simple asymptotic results for this outage performance in high SNR regime are provided, from which the achieved diversity and coding gains and the diversity-multiplexing trade-off can be extracted. In addition, to further enhance the secondary network, optimal power allocation among hops is obtained based on the asymptotic outage performance under the constraints of transmit power of a secondary transmit station and interference limit on the primary network. The developed analytical results in this work are validated through numerical and simulation results.
\end{abstract}

Index Terms-Cognitive radio, MIMO, Multihop, relaying, interference cancellation, spectrum sharing, outage probability.

\section{INTRODUCTION}

A key advantage of multihop wireless networks is their ability to connect a transmit source to a destination through multiple hops, thereby expanding the network coverage while alleviating the need for fixed infrastructure $[1,2]$. In addition, cognitive relaying networks (CRNs) are considered as one of the promising solutions for radio spectrum scarceness and bandwidth limitations. They can significantly improve the spectral efficiency of wireless networks by intelligently sharing

F. Al-Qahtani, and S. Hessien, and H. Alnuweiri are with Electrical \& Computer Engineering Program, Texas A\&M University at Qatar, Doha 23874, Qatar E-mail: \{fawaz.al-qahtani, salah.hessien hussein.alnuweiri\}@qatar.tamu.edu. R. M. Radaydeh is is with Computer, Electrical, and Mathematical Sciences and Engineering Division, King Abdullah University of Science and Technology (KAUST), Thuwal, 23955-6900, KSA, E-mail: redha.radaydeh@gmail.com, and and T. Q. Duong is with Queen's University Belfast, Belfast BT7 1NN, U.K. e-mail: trung.q.duong@qub.ac.uk.

This paper was made possible by NPRP grant No. 8-1545-2-657 from the Qatar National Research Fund (a member of Qatar Foundation). The statements made herein are solely the responsibility of the authors. spectrum resources [3]-[5]. A common approach in spectrum sharing network is the underlay cognitive radio model in which the secondary user (SU) is allowed to share the spectrum with the primary user (PU) simultaneously under an interference limit to maintain a reliable operation of the primary network.

Due to their promising advantages, underlay cognitive networks with relaying have gained research interests in recent years (see [7]-[10] and references therein). Specifically, in [7], the outage probability of spectrum sharing decode-andforward (DF) relaying with the best relay selection in Rayleigh fading was investigated, which was extended to Nakagami- $m$ fading in [8]. In [9], the authors investigated the impact of PU interference on the outage performance of cognitive DF relaying in Rayleigh fading. In [10], the outage performance of dual-hop DF with spectrum sharing in the presence of the direct link was investigated in Nakagami- $m$ fading. The outage probability of dual-hop amplify-and-forward (AF) in Rayleigh fading was examined in [11]. Moreover, the work in [12] investigated the impact of multiple PUs on the outage probability of dual-hop DF relaying, which was extended to multihop CRNs in [13]-[15].

To further improve the performance of CRNs, some works have incorporated multiple-input multiple-output (MIMO) techniques [16-19]. In particular, transmit antenna selection with receive maximal ratio combining (TAS/MRC) has been widely adopted as a low complexity and power efficient approach which achieves full diversity gain [20], [21]. However, the advantages of TAS/MRC are limited by several factors, such as the spacing between transmit and receive antennas, and accuracy of channel estimation, and the accuracy of feedback channel for TAS implementation [22]. Moreover, when receive antennas are highly correlated due to space limitation at the receive station, the use of receive MRC becomes a waste of processing as no diversity gain can be achieved. In this regard, the use of smart arrays can be of interest to mitigate the effect PU interference on the secondary network [23]. Specifically, with the knowledge of the direction of arrival (DoA) of interference sources, the receive array radiation pattern can be shaped to place deep nulls in the directions of some resolvable interference sources [26], while providing a maximum array gain in the direction of the desired signal. This interference cancellation mechanism can have real applications in multihop CRNs as the mitigation of interference on the secondary network can be of prime importance when primary PUs access the shared spectrum resources heavily. Two interference cancellation algorithms were proposed; namely dominant receive interference cancellation (DRIC) [27], [28], 
and adaptive receive interference cancellation (ARIC) [29]. The first algorithm requires perfect predication and statistical ordering of interference powers, whereas the latter does not need prior knowledge of the statistical properties of interference sources and can efficiently utilize the available receive antennas.

The aforementioned IC algorithms do not provide any receive diversity gain; in contrast to receive MRC when receive antennas are sufficiently spaced, but they are preferable for cases when receive antennas are insufficiently spaced and/or the system undergoes deep interference effect. One of the main objectives of this paper is to provide detailed analytical treatments that enable a comprehensive comparison of the achieved performance of underlay cognitive secondary network with AF multihop relaying under various MIMO scenarios for different receive arrays operation per hop. Specifically, the developed results characterizes the use of the conventional TAS/MRC per hop when the receive antennas per hop are sufficiently spaced, and allows for insightful treatment against TAS/DRIC and/or TAS/ARIC when receive diversity can not be achieved.

The contributions of this paper are summarized as follows:

- We treat a generalized model of that adopted in [13][15]. Specifically, MIMO AF multihop underlay CRNs is considered with the use of TAS/MRC per hop in the secondary network in the presence of multiple PUs and under the effect of Rayleigh fading. The model is particularly applicable when the spacing between receive antennas per hop are sufficient to exploit diversity through MRC. The corresponding end-to-end signal-tointerference-plus-noise ratio (SINR) outage probability is obtained in closed-form. In addition, the asymptotic results for this outage performance in high SNR regime are obtained, which are found useful to predict the achieved diversity and coding gains, and characterize the diversity-multiplexing trade-off (DMT) of the considered system.

- The analysis is then extended in order to treat two new models for TAS/IC per hop in the secondary network of AF multihop underlay CRNs, which are TAS/DRIC and TAS/ARIC. These models are useful when receive diversity can not be achieved with the use of MRC and the effect of primary network interference on each hop of the secondary network is significant. For these models, the end-to-end SINR statistics are characterized, which are then used to obtain new results for the end-to-end outage probability of the secondary network. Moreover, simple results for the asymptotic outage performance are derived for the two models, which are then used to predict the achieved diversity and coding gains, and characterize the achieved DMT.

- To further enhance the performance of the TAS/MRC model or TAS/IC models, the optimal solutions for the power allocation to maximize the outage performance under constraints of transmit power of a secondary transmit station and interference limit on the primary network are obtained.

Based on the developed results for the various schemes men- tioned above, the following outcomes are noted:

- TAS/MRC scheme is shown to outperform TAS/DRIC and TAS/ARIC in high average SNR/SINR regime, assuming that maximum diversity gain is achievable from MRC. However, even with maximized receiver MRC gain, it is observed that TAS/IC schemes outperforms TAS/MRC in low SNR/SINR regime. Thus, TAS/DRIC and TAS/ARIC can be particularly useful under deep effect of primary network interference.

- The asymptotic results for end-to-end outage performance of different schemes show that the achieved diversity gain for TAS/MRC scheme with sufficiently spaced receive antennas is $N_{S} N_{R}$ per hop, while the diversity gain of both TAS/IC schemes is $N_{S}$ as well as TAS/MRC with insufficiently spaced antennas per hop, where $N_{S}$ and $N_{R}$ are the sizes of transmit and receive arrays per hop, respectively. This outcome is observed when the interference power limit $\mathcal{Q}_{I}$ is proportional to the maximum transmit power $\mathcal{Q}_{P}$. However, when the interference power limit is fixed, an outage floor appears, and hence zero diversity gain for TAS/MRC and TAS/IC schemes is noticed. Herein, TAS/DRIC and TAS/ARIC can have another practical advantage as compared to TAS/MRC in mitigating the effect of primary network interference, and hence boost the end-to-end outage performance of the secondary network.

- The results indicate that TAS/DRIC outperforms TAS/ARIC under deep effect of interference from primary network per hop due to the variations of the achieved coding gains of these two schemes, but at the expense of complicated processing and degraded operation efficiency.

- It is noted that the increase in the number of PUs transmit and receive antennas and number of hops in the secondary network do not affect the achieved diversity gains of TAS/MRC and TAS/IC schemes. However, they degrade the coding gains of these schemes differently.

The rest of the paper is organized as follows. The adopted system and channel models are discussed in Section II. Sections III and IV present the signal model and analytical performance results of the considered system without IC, respectively. Sections V and VI address the signal model and performance analysis under different receive algorithms IC, respectively. Section VII presents some numerical results that are supported by simulations. Finally, Section VIII contains concluding remarks.

Mathematical Notations: The notations used herein are as follows. Bold lower/upper case symbols denote vectors/matrices, respectively, $(\cdot)^{T}$ for transpose, $(\cdot)^{H}$ for conjugate transpose, and $|\cdot|$ and $\|\cdot\|_{F}$ are used for the absolute value and the Frobenious norm of vectors/matrices, respectively. The term $f_{\beta}(x)$ is the probability distribution function (PDF) of random variable (RV) $\beta$, and $F_{\beta}(x)$ is its cumulative distribution function (CDF), and $\mathcal{R} e\{t\}$ is the real part of a complex quantity $t$. The function $\Gamma(\cdot, \cdot)$ is the upper-incomplete gamma function, which is defined as $\Gamma(z, x)=\int_{x}^{\infty} t^{z-1} e^{-t} \mathrm{dt}$, and $\gamma(z, x)=\int_{0}^{x} t^{z-1} e^{-t} \mathrm{dt}$ is the 


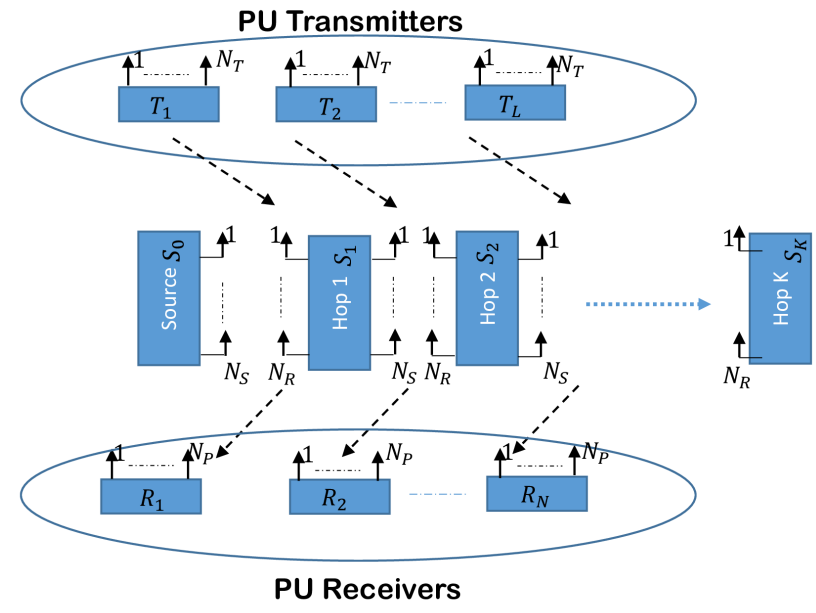

Fig. 1. Adopted system model.

lower-incomplete gamma function.

\section{System And Channel Models}

The system model under consideration as depicted in Fig. 1 treats a generalization model for underlay CRN that consists of a secondary network and a primary network. The secondary network is allowed to access the same spectrum resources of the primary network under certain interference limit condition. It aims to establish a cooperative path between a desired source and a desired destination. Due to the absence of direct link between these two stations, multihop relaying is adopted which contains a total of $K$ relaying hops to realize the intended coverage between the source and destination. Therefore, this relaying model leads to the degraded transmission efficiency due to the use of multiple time slots to establish the intended relaying path for extended coverage.

The secondary network of interest contains a total of $(K+1)$ nodes, which are denoted as $\left\{S_{0}, S_{1}, \ldots, S_{K}\right\}$, wherein $S_{0}$ and $S_{K}$ refer to the desired source and destination, respectively. The same information from $S_{0}$ is relayed through AF protocol over successive time slots to reach the destination $S_{K}$, wherein the primary network spectrum resources can be reused in each relaying hop. Each relaying node is equipped with $N_{S}$ transmit antennas at one side and $N_{R}$ receive antennas at the other side. The receive antennas at each relaying node can be either sufficiently spaced, wherein MRC can provide the maximum possible receive diversity gain, or insufficiently spaced, wherein IC can be useful to alleviate interference when MRC is useless. However, its transmit antennas are considered to be sufficiently spaced throughout this paper ${ }^{1}$.

The effectiveness of TAS per hop is related to several factors, such as the accuracy of per hop channel estimation, efficiency of feedback channel, and accuracy of hardware processing for best possible transmit antenna selection per hop [21], [22]. In this paper, and for the sake of brevity, we adopt an ideal TAS implementation that guarantees best

\footnotetext{
${ }^{1}$ The consideration of possible transmit correlation among transmit antennas with TAS or receive antennas with MRC can be also incorporated into the analysis, and it will be a topic of future work on the scope of this paper.
}

possible transmit antenna selection per hop, but the considerations of the aforementioned issues can be incorporated into the developed models. Moreover, a quasi-static channel model per hop is adopted, in which the transmit selection based on large-scale fading will be useless. Herein, the gains of transmit antenna selection per hop will be noticeable under TAS based on quasi-static small-fading, which can be feasible outdoors.

It is considered that the primary network contains a number of $L$ PU transmit stations, which are denoted as $\left(T_{1}, \ldots, T_{L}\right)$, and a number of $N$ PU receive stations, refereed to as $\left(R_{1}, \ldots, R_{N}\right)$, wherein each $\mathrm{PU}$ transmit and receive stations are equipped with $N_{T}$ and $N_{P}$ number of antennas, respectively. The PU transmit stations can transmit information over the successive time slots of the relays in the secondary network, and hence, they are likely to induce independent interference sources at each receive relay station in the secondary network, wherein each interference source will be observed over the $N_{R}$ antennas of the relay station. Moreover, a transmit relay station is expected to induce interference at PU receive stations during its transmission time, wherein such interference source will be observed at the $N_{P}$ antennas of each PU receive station in an independent manner among different PU receive stations. This interference power is linked to the allowed transmit power level at that relay station.

The channel gains on the $S_{k-1} \rightarrow S_{k}$ relaying hop of the secondary network are denoted by $h_{k i j}$, for $i \in\left\{1, \ldots, N_{S}\right\}$ and $j \in\left\{1, \ldots, N_{R}\right\}$. Moreover, the channel gains from the $i$ th transmit antenna of the relay node $S_{k}$ in the secondary network to the $n$th PU receive station are referred to as $\mathbf{g}_{k i n}=\left\{g_{k i n 1}, \ldots, g_{k i n N_{P}}\right\}$ for $i \in\left\{1, \ldots, N_{S}\right\}$ and $n \in\{1, \ldots, N\}$. In addition, the channel gains of interference sources observed from the $L$ PU transmit stations at relay node $S_{k}$ are referred to as $\mathbf{f}_{k l q}=\left\{f_{k l q 1}, \ldots, f_{k l q N_{R}}\right\}$, for $l \in\{1, \ldots, L\}$ and $q \in\left\{1, \ldots, N_{T}\right\}$. Perfect channel estimation of all associated channel gains are assumed per each hop of the secondary network as well as at PU stations, and they are normalized to have independent and identical distribution (i.i.d) complex-valued Gaussian distributions. However, these assumption can be relaxed, but at the expense of more involved analytical treatments.

\section{Signal Models AND End-TO-End SINR Without RECEIVE INTERFERENCE CANCELLATION}

Considering the $k$ th hop of the secondary network, the relay station $S_{k}$, receives on its array of size $N_{R}$, a faded version of the data signal from the transmit relay station $S_{k-1}$, which is corrupted by $L$ faded and independent interference signals from the active $L \mathrm{PU}$ transmit stations. Conditioned on the event that the transmit node $S_{k-1}$ uses its $i$ th transmit antenna at that time, for $i=1,2, \ldots, N_{S}$, the received signal vector at $S_{k}$ is given by

$\mathbf{y}_{k i}=\sqrt{P_{k-1} \Omega_{k, h}} \mathbf{h}_{k i} x_{k-1}+\sqrt{P_{T} \Omega_{k, f}} \sum_{l=1}^{L} \mathbf{f}_{k l q} \hat{x}_{k-1, l}+\mathbf{n}_{k}$

where $\mathbf{y}_{k i}=\left[y_{k i 1}, \ldots, y_{k i N_{R}}\right]$ is a $1 \times N_{R}$ vector of received signals on the $N_{R}$ receive antennas at $S_{k}, P_{k-1}$ is the transmit 
power at $S_{k-1}$ node, $x_{k-1}$ is the desired transmitted symbol of normalized power, $P_{T}$ is the transmit power per PU transmit node, $\hat{x}_{k-1, l}$ is the transmitted symbol from the $l$ th PU transmit station, $\mathbf{h}_{k i}=\left[h_{k i 1}, \ldots, h_{k i N_{R}}\right]$ is an $1 \times N_{R}$ vector of channel gains between the $i$ th transmit antenna at $S_{k-1}$ and the $N_{R}$ receive antennas at $S_{k}, \Omega_{k, h}$ is the average fading power on the $k$ th hop from $S_{k-1}$ to $S_{k}$. Moreover, the term $\mathbf{f}_{k l q}=\left[f_{k l q 1}, \ldots, f_{k l q N_{R}}\right]$ in the preceding result refers to a $1 \times N_{R}$ vector of the same channel gain (i.e., $f_{k l q j}=f_{k l q}$, for $j=1,2, \ldots, N_{R}$ ) that is associated with the interference source originated by the $l$ th PU transmit node equipped with $N_{T}$ antennas and observed at the $S_{k}$ relay receive node, and $\Omega_{k, f}$ is the average fading power of the interference source due to PU transmit station and observed at $S_{k}$. The term $\mathbf{n}_{k}=\left[n_{k 1}, \ldots, n_{k N_{R}}\right]$ is a $1 \times N_{R}$ vector of uncorrelated samples of additive white Gaussian noise (AWGN) at $S_{k}$, where each of which has zero mean and $\sigma^{2}$ average power. The transmitted symbols in primary and secondary networks at any time instant are normalized to have zero-mean and unity variance. The transmission interval per hop is assumed to be equal for all time slots.

To maintain the successful operation of the secondary network with full access to the spectrum resources of the primary network per each hop, it is required that the interference power levels affecting the $N$ PU receive stations and originated due to each hop transmission in the secondary network must not exceed a predetermined threshold level $I_{P}$. Therefore, to protect the primary network against interference sources from the secondary network, the transmit power per hop in the secondary network can be adjusted following the model in [3], [5], which gives

$$
P_{k}=\min \left\{P_{s}, \frac{I_{P}}{\lambda_{k, g}}\right\}, \quad k=0,1, \ldots, K-1
$$

where $I_{P}$ is the maximum allowed interference power per PU receive station and $P_{s}$ is the peak transmit power per transmit relay in the secondary network. Conditioned on the event that the $i$ th transmit antenna is used at the transmit relay, say $S_{k}$ node, the vector $\mathbf{g}_{k i n}=\left[g_{k i n 1}, \ldots, g_{k i n N_{P}}\right]$ refers to the same interference channel gain of an average fading power $\Omega_{k, g}$, which is observed at the $n$th PU receive station (i.e., $g_{k i n t}=g_{k i n}$, for $t=1,2, \ldots, N_{P}$ ). Moreover, to account for the maximized effect of interference at PU receive stations due to the transmission by $S_{k}$ node, it is reasonable to adjust the term $\lambda_{k, g}$ in the preceding result as $\lambda_{k, g}=\max _{1 \leq n \leq N}\left\{\left|g_{k i n}\right|^{2}\right\}$, irrespective of the transmit antenna index used in the secondary network per the $k$ th hop. This refers to that case that the transmit power at $S_{k}$ is adjusted relative to its maximized interference effect at the $N$ PU receive stations.

For the AF relying protocol in the secondary network, the $k$ th relay $S_{k}$ amplifies its received signal by a gain $A_{k}$ and transmits the amplified signal $\mathbf{z}_{k}=A_{k} \mathbf{y}_{k}$ in the next time slot. For simplicity, it is considered that the power constraint at the $k$ th relay is satisfied when $A_{k}=\sqrt{\frac{P_{k}}{P_{k-1} \lambda_{k, h}+\sigma^{2}}}$ is applied. Therefore, the end-to-end SINR signal model at the destination with use of AF protocol can be expressed as shown in (3) on the next page. Then, as per the results in [1], the end-to-end SINR of the secondary network can be written as

$$
\begin{aligned}
\gamma_{\mathrm{E} 2 \mathrm{E}} & =\left[\prod_{k=1}^{K}\left(1+\frac{1+\gamma_{k, \mathrm{I}}}{\frac{P_{k} \lambda_{k, h}}{\sigma^{2}}}\right)-1\right]^{-1}, \\
& \leq \min \left\{\gamma_{1}, \gamma_{2}, \ldots, \gamma_{K}\right\} .
\end{aligned}
$$

where $\gamma_{k} \triangleq \min \left(\mathcal{Q}_{P}, \frac{\mathcal{Q}_{I}}{\lambda_{k, g}}\right) \frac{\lambda_{k, h}}{1+\gamma_{k, \mathrm{I}}}$. The result of $\gamma_{k}$ follows from (4) after using the results in (1) and (2), respectively. Herein, $\mathcal{Q}_{P} \triangleq P_{s} / \sigma^{2}$ and $\mathcal{Q}_{I} \triangleq I_{P} / \sigma^{2}$. In addition, the term $\lambda_{k, h}$ is obtained as follows. The use of the receive MRC at the receive relay $S_{k}$, conditioned on the $i$ th transmit antenna of the transmit relay $S_{k-1}$, results in $\left\|\mathbf{h}_{k i}\right\|^{2}=\sum_{j=1}^{N_{R}}\left|h_{k i j}\right|^{2}$, for $i=1,2, \ldots, N_{S}$. Therefore, the MRC gain is observed under the condition that the $N_{R}$ receive antennas at $S_{k}$ are sufficiently spaced to provide uncorrelated copies of the same faded data symbol on its receive array. Then, to extract the benefits of the $N_{S}$ transmit antennas at $S_{k-1}$, the node $S_{k}$ predicts the index of the transmit antenna at $S_{k-1}$ to be used for subsequent transmission, which will maximize the combined fading power $\left\|\mathbf{h}_{k i}\right\|^{2}$. Under perfect TAS, the term $\lambda_{k, h}$ in $\gamma_{k}$ can be then expressed as $\lambda_{k, h}=\max _{1 \leq i \leq N_{S}}\left\{\left\|\mathbf{h}_{k i}\right\|^{2}\right\}$, which refers to the combined desired power per hop in the secondary network. Moreover, the term $\gamma_{k, \mathrm{I}}$ in $\gamma_{k}$ refers to the aggregate interference-to-noise ratio (INR) per hop in the secondary network. With the use of receive antennas for MRC, interference sources observed from the $L$ PU transmit stations can not be mitigated. Therefore, this term can be expressed as $\gamma_{k, \mathrm{I}}=\sum_{l=1}^{L} \bar{\gamma}_{k, \mathrm{I}}\left|f_{k l q}\right|^{2}$, where $\bar{\gamma}_{k, \mathrm{I}} \triangleq P_{T} \Omega_{k, f} / \sigma^{2}$ is the average INR per interference source. The PDF of $\gamma_{k, \mathrm{I}}$ can be now expressed as

$$
f_{\gamma_{k, \mathrm{I}}}(x)=\frac{1}{\Gamma\left(L_{T}\right)}\left(\frac{1}{\bar{\gamma}_{k, \mathrm{I}}}\right)^{L_{T}} x^{L_{T}-1} \exp \left(-\frac{x}{\bar{\gamma}_{k, \mathrm{I}}}\right) .
$$

where $L_{T}=L N_{T}$. Based on the preceding results, the statistics of $\lambda_{k, g}$ and $\lambda_{k, h}$ can be obtained as follows. The PDF and CDF of $\lambda_{X}=\left\{\lambda_{k, h}, \lambda_{k, g}\right\}$ with $\mathcal{N}=\left\{N_{S}, L_{P}\right\}$, $L_{P}=N_{P} N, \mathcal{Y}=\left\{N, N_{R}\right\}$ and $\Omega_{X}=\left\{\Omega_{k, h}, \Omega_{k, g}\right\}$ are given respectively by [12]

$$
\begin{aligned}
F_{\lambda_{X}}(x) & =\left(1-e^{-\frac{x}{\Omega_{X}}} \sum_{p=1}^{\mathcal{Y}} \frac{1}{\Gamma(p)}\left(\frac{x}{\Omega_{X}}\right)^{p-1}\right)^{\mathcal{N}} \\
& =1-\sum_{k_{1}=1}^{\mathcal{N}}\left(\begin{array}{l}
\mathcal{N} \\
k_{1}
\end{array}\right)(-1)^{k_{1}-1} \Gamma\left(k_{1}+1\right) \\
& \sum_{\left|\mathbf{c}_{\mathbf{p}}\right|=k_{1}} \mu_{p}\left(\frac{x}{\Omega_{X}}\right)^{\lambda_{p}} \exp \left(-\frac{k_{1} x}{\Omega_{X}}\right),
\end{aligned}
$$




$$
\begin{aligned}
\mathbf{Y}_{K} & =\sqrt{P_{K-1} \Omega_{K, h}} \mathbf{h}_{K i} \prod_{k=1}^{K-1} A_{k} \sqrt{P_{k} \Omega_{k, h}} \mathbf{h}_{k i} x_{k}+\sqrt{P_{T} \Omega_{K, f}} \sum_{l=1}^{L} \mathbf{f}_{K l q} \hat{x}_{K, l}+\mathbf{n}_{K} \\
& +\sum_{k=1}^{K-1}\left(\prod_{n=k}^{K-1} A_{n} \sqrt{P_{n} \Omega_{n, h}} \mathbf{h}_{n i}\right) \sqrt{P_{T} \Omega_{k, f}} \sum_{l=1}^{L} \mathbf{f}_{k l q} \hat{x}_{k, l}+\sum_{k=1}^{K-1}\left(\prod_{n=k}^{K-1} A_{n} \sqrt{P_{n} \Omega_{n, h}} \mathbf{h}_{n i}\right) \mathbf{n}_{k}
\end{aligned}
$$

and

$$
\begin{array}{r}
f_{\lambda_{X}}(x)=\frac{\mathcal{N}}{\Gamma\left(N_{R}\right) \Omega_{X}} \sum_{k_{4}=0}^{\mathcal{N}-1}\left(\begin{array}{c}
\mathcal{N}-1 \\
k_{4}
\end{array}\right)(-1)^{k_{4}} \Gamma\left(k_{4}+1\right) \\
\sum_{\left|\mathbf{c}_{\mathbf{n}}\right|=k_{4}} \mu_{n}\left(\frac{x}{\Omega_{X}}\right)^{\lambda_{n}+N_{R}-1} \exp \left(-\frac{\left(k_{4}+1\right) x}{\Omega_{X}}\right)
\end{array}
$$

where $\mathbf{c}_{\mathbf{n}}=\left(c_{1}, \ldots, c_{\mathcal{T}}\right) \in \mathcal{T}^{\mathcal{T}}$ is a multi index $\mathcal{T}$-tuple vector of nonnegative integers of length $\left|\mathbf{c}_{\mathbf{n}}\right|=\sum_{n=1}^{\mathcal{T}} c_{n}=k_{4}$ and $\mu_{n}=\frac{1}{\prod_{n=1}^{\mathcal{N}} \Gamma\left(c_{n}+1\right) \Gamma(n)^{c_{n}}}$ and $\lambda_{n}=\sum_{n=1}^{\mathcal{N}} c_{n}(n-1)$.

Proof: The derivations of (6) and (8) are obtained by applying the binomial expansion in [33, Eq.1.111] and the power sum expansion of [32, Eq. 9].

\section{End-TO-End OUtAge Probability Without RECEIVE INTERFERENCE CANCELLATION}

This section builds on the descriptions provided in section III, and investigates the outage probability of the underlay cognitive MIMO system with AF multihop in the secondary network. It considers the TAS/MRC scheme without receive interference cancellation per hop, but in the presence of interference from PU transmit stations, as described in section III. The section contains three main parts, wherein the first two parts develop new analytical results for the exact and asymptotic end-to-end outage probability, and the last part treats the optimal power allocation problem per hop to further improve the achieved performance of the secondary network.

\section{A. Exact Analysis of Outage Probability}

The outage probability is an important performance indicator of wireless systems, which is defined as the probability that the end-to-end SINR falls below a specific threshold $\gamma_{\mathrm{th}}$. Based on (4), it can be expressed as

$$
P_{\text {out }}\left(\gamma_{\text {th }}\right)=1-\prod_{k=1}^{K}\left(1-F_{\gamma_{k}}\left(\gamma_{\text {th }}\right)\right) \text {. }
$$

We present a new closed-form expression for the end-to-end SINR outage probability in the following key result.

Theorem 1: The end-to-end SINR $\gamma_{\mathrm{E} 2 \mathrm{E}}$ outage probability of underlay cognitive system with AF multihop relaying and TAS/MRC per hop is upper bounded by (9), where $F_{\gamma_{k}}\left(\gamma_{\mathrm{th}}\right)$ is given by (10) as shown on the top of the next page, where $\phi=\frac{k_{1} \gamma_{\mathrm{th}}}{\bar{\gamma}_{k, h} \mathcal{Q}_{I}}+\frac{\left(k_{3}+1\right)}{\Omega_{k, g}}, \mu=\lambda_{p}+\lambda_{l}+N, \mu_{2}=\lambda_{l}+N$, $\lambda_{l}=\sum_{l=1}^{N} c_{l}(l-1)$ and $U($.$) is a confluent hypergeometric$ function [33, 9.210.2].

Proof: See Appendix A.
Note that Theorem 1 involves standard functions and a special confluent hypergeometric function. This allows for fast evaluation in popular mathematical software such as Matlab, which provides an efficient means to compute the outage probability analytically, thereby avoiding the need for timeconsuming Monte Carlo simulations.

\section{B. Asymptotic Analysis}

In this subsection, we derive the outage probability in the asymptotic regime based on which the diversity and coding gains achieved by the system are investigated. We assume herein two practical scenarios: 1) proportional interference power constraint, where the peak interference power is proportional to the maximum transmit power such that $\mathcal{Q}_{I}=\mu \mathcal{Q}_{P}$ as $\mathcal{Q}_{P} \rightarrow \infty$, where $\mu$ is a positive constant [8], and 2) fixed interference power constraint, where the peak interference power is fixed and independent of the maximum transmit power as $\mathcal{Q}_{P} \rightarrow \infty$ [11].

1) Proportional interference power constraint: This scenario considers that $\mathrm{PU}$ receive stations can tolerate high level of interference from SU transmit stations. Hence, we present the following key result.

Proposition 1: The asymptotic outage probability of underlay cognitive system with AF multihop relaying and TAS/MRC per hop, under the condition that $\mathcal{Q}_{I}=\mu \mathcal{Q}_{P}$ as $\mathcal{Q}_{P} \rightarrow \infty$, is given by

$$
P_{\mathrm{out}}^{\infty}\left(\gamma_{\mathrm{th}}\right) \approx \sum_{k=1}^{K} \Psi_{k}\left(\frac{\gamma_{\mathrm{th}}}{\mathcal{Q}_{P}}\right)^{N_{S} N_{R}}
$$

where $\Psi_{k}$ is given in (12) as shown on the next page, in which $t_{1}=N_{S} N_{R}+\lambda_{l}+N$.

Proof: See Appendix B.

Based on Proposition 1, we present the following corollaries.

Corollary 1: The diversity and coding gains of underlay cognitive system with AF multihop relaying and TAS/MRC per hop, under the condition that $\mathcal{Q}_{I}=\mu \mathcal{Q}_{P}$ as $\mathcal{Q}_{P} \rightarrow \infty$, are given respectively by $\mathcal{G}_{D}=N_{S} N_{R}$ and

$$
\mathcal{G}_{C}=\min _{k=1 \ldots K} \frac{\left(\Psi_{k}\right)^{-\frac{1}{N_{S} N_{R}}}}{\gamma_{\mathrm{th}}} .
$$

Proof: It follows by re-expressing the asymptotic outage probability in Proposition (1) as $P_{\text {out }}^{\infty} \approx\left(\mathcal{G}_{C} \mathcal{Q}_{P}\right)^{-\mathcal{G}_{D}}$.

Corollary 2: The DMT of underlay cognitive system with AF multihop relaying and TAS/MRC per hop, under the condition that $\mathcal{Q}_{I}=\mu \mathcal{Q}_{P}$ as $\mathcal{Q}_{P} \rightarrow \infty$, is given by

$$
d(\hat{r})=(1-2 \hat{r}) N_{S} N_{R} .
$$




$$
\begin{aligned}
& F_{\gamma_{k}}\left(\gamma_{\mathrm{th}}\right)=1-\frac{1}{\Gamma\left(L_{T}\right)}\left(\frac{1}{\bar{\gamma}_{k, \mathrm{I}}}\right)^{L_{T}} \sum_{k_{1}=1}^{N_{S}}\left(\begin{array}{c}
N_{S} \\
k_{1}
\end{array}\right)(-1)^{\left(k_{1}-1\right)} \Gamma\left(k_{1}+1\right) \exp \left(-\gamma_{\mathrm{th}}\left(\frac{k_{1}}{\Omega_{k, h} Q_{P}}\right)\right) \sum_{\left|\mathbf{c}_{\mathbf{p}}\right|=k_{1}} \mu_{p}\left(\frac{\gamma_{\mathrm{th}}}{\Omega_{k, h} Q_{P}}\right)^{\lambda_{p}} \\
& \quad \times \sum_{v=0}^{\lambda_{p}}\left(\begin{array}{c}
\lambda_{p} \\
v
\end{array}\right) \Gamma(L+v)\left(\frac{k_{1} \gamma_{\mathrm{th}}}{\Omega_{k, h} Q_{P}}+\frac{1}{\bar{\gamma}_{k, \mathrm{I}}}\right)^{-\left(L_{T}+v\right)}+\frac{L_{T}}{\Gamma(N) \Omega_{k, g}} \sum_{k_{1}=1}^{N_{S}}\left(\begin{array}{c}
N_{S} \\
k_{1}
\end{array}\right)(-1)^{k_{1}-1} \Gamma\left(k_{1}+1\right) \\
& \quad \times \sum_{\left|\mathbf{c}_{\mathbf{p}}\right|=k_{1}} \mu_{p} \sum_{k_{3}=0}^{L-1}\left(\begin{array}{c}
L_{T}-1 \\
k_{3}
\end{array}\right)(-1)^{k_{3}} \Gamma\left(k_{3}+1\right) \sum_{\left|\mathbf{c}_{\mathbf{l}}\right|=k_{3}} \mu_{l}\left(\frac{1}{\Omega_{k, g}}\right)^{\lambda_{l}+N-1}\left(\frac{\gamma_{\mathrm{th}}}{\Omega_{k, h} \mathcal{Q}_{I}}\right)^{\lambda_{p}} e^{-\frac{Q_{I}}{Q_{P}} \phi} \sum_{k=0}^{\mu-1} \frac{\Gamma(\mu)}{\Gamma(k+1)}\left(\frac{Q_{I}}{Q_{P}}\right)^{k} \\
& \quad \times\left(\frac{1}{\phi}\right)^{\mu-k} \frac{1}{\Gamma\left(L_{T}\right)}\left(\frac{1}{\bar{\gamma}_{k, \mathrm{I}}}\right)^{L_{T}} \sum_{\zeta=0}^{\lambda_{p}}\left(\begin{array}{c}
\lambda_{p} \\
\zeta
\end{array}\right) \frac{\Gamma\left(\zeta+L_{T}\right)}{\left(\frac{\phi \Omega_{k, h} \mathcal{Q}_{I}}{k_{1} \gamma_{\mathrm{th}}}\right)^{-\left(L_{T}+\zeta\right)}} U\left(\zeta+L, \zeta+L-\mu+k+1, \frac{\phi}{k_{1} \theta}\left(\frac{Q_{I} k_{1} \theta}{Q_{P}}+\frac{\gamma_{\mathrm{th}}}{\mathcal{Q}_{I} \Omega_{k, h} \bar{\gamma}_{k, \mathrm{I}}}\right)\right),
\end{aligned}
$$

$$
\begin{aligned}
\Psi_{k} & =\left(\frac{1}{\bar{\gamma}_{k, \mathrm{I}}}\right)^{L_{T}}\left(\frac{1}{\Gamma\left(N_{R}+1\right)}\right)^{N_{S}}\left(\frac{1}{\Omega_{k, h}}\right)^{N_{S} N_{R}} U\left(L, N_{S} N_{R}+L_{T}+1, \frac{1}{\bar{\gamma}_{k, \mathrm{I}}}\right)\left(\mu^{-N_{S} N_{R}} F_{\lambda_{k, g}}\left(\frac{Q_{I}}{Q_{P}}\right)\right. \\
& \left.+\frac{L_{T}}{\Gamma(N)}\left(\frac{\Omega_{k, g}}{Q_{I}}\right)^{N_{S} N_{R}} \sum_{k_{3}=0}^{L-1}\left(\begin{array}{c}
L_{T}-1 \\
k_{3}
\end{array}\right)(-1)^{k_{3}} \Gamma\left(k_{3}+1\right) \sum_{\left|\mathbf{c}_{\mathbf{1}}\right|=k_{3}} \mu_{l}\left(k_{3}+1\right)^{-t_{1}} \Gamma\left(t_{1}, \frac{Q_{I}\left(k_{3}+1\right)}{Q_{P} \Omega_{k, g}}\right)\right)
\end{aligned}
$$

where $\hat{r}=\frac{R}{K \log _{2}\left(1+\mathcal{Q}_{P}\right)}$ is the normalized spectral efficiency with respect to the channel capacity, and $R$ is the spectral efficiency in bits/s/ $\mathrm{Hz}$.

Proof: See Appendix C.

From Proposition 1, we observe the intuitive result that, when the normalized spectral efficiency $\hat{r} \rightarrow 0$, the maximum diversity order of $N_{S} N_{R}$ is achieved. While for the case when $d(\hat{r}) \rightarrow 0$, the maximum normalized spectral efficiency of $\frac{1}{K}$ can be obtained. This implies that the DMT is entirely dependent on the secondary network, and independent of the primary network.

2) Fixed interference power constraint: This scenario considers $\mathcal{Q}_{I}$ is fixed, and $\mathcal{Q}_{P}$ grows large in the high SNR. For such scenario, we present the following key result.

Proposition 2: The asymptotic outage probability of underlay cognitive system with AF multihop relaying and TAS/MRC per hop, under the condition that $\mathcal{Q}_{I}$ is fixed as $\mathcal{Q}_{P} \rightarrow \infty$, is given by

$$
P_{\text {out }}^{\infty}\left(\gamma_{\mathrm{th}}\right) \approx \frac{\sum_{k=1}^{K}\left(\Phi_{k, 1}\left(\frac{1}{\mathcal{Q}_{P}}\right)^{N_{S} N_{R}}+\Phi_{k, 2}\left(\frac{1}{\mathcal{Q}_{I}}\right)^{N_{S} N_{R}}\right)}{\gamma_{\mathrm{th}}^{-N_{S} N_{R}}}
$$

where $\Phi_{k, 1}$ and $\Phi_{k, 2}$ are given respectively by (16) and (17) on the next page.

Proof: It follows the proof of Proposition 1.

Corollary 3: The diversity and coding gains of underlay cognitive system with AF multihop relaying and TAS/MRC per hop are given respectively by $\mathcal{G}_{D}=N_{S} N_{R}$ and

$$
\mathcal{G}_{C}=\min _{k=1 \ldots K} \frac{\Phi_{k, 1}^{-\frac{1}{N_{S}}}+\left(\Phi_{k, 1} \mu\right)^{-\frac{1}{N_{S}}}}{\gamma_{\mathrm{th}}} .
$$

\section{Optimal Power Allocation}

This part addresses the optimum power allocation for the end-to-end outage probability of the considered underlay cognitive system with AF multihop relaying and TAS/MRC per hop. In particular, we choose the outage probability of secondary network as our objective function, and formulate an optimization problem by considering total power constraint $P_{\text {tot }}=\sum_{k=1}^{K} P_{k}$ in the secondary network as well as peak interference power limit. For simplicity, the asymptotic outage probability of the secondary network under proportional interference power constraint is considered. Hence, the objective function of the optimization problem becomes

$$
\begin{aligned}
& \min P_{\text {out }}^{\infty}\left(\gamma_{\mathrm{th}}\right)=\sum_{k=1}^{K} \Psi_{k}\left(\frac{\gamma_{\mathrm{th}}}{P_{k}}\right)^{N_{S} N_{R}} \\
& \text { subject to }\left\{\begin{array}{l}
\sum_{k=1}^{K} P_{k} \leq P_{\mathrm{tot}}, \\
P_{k} \leq P_{s}, \\
\sum_{k=1}^{K} \lambda_{k, g} P_{k} \leq I_{P},
\end{array}\right.
\end{aligned}
$$

It can be observed that the asymptotic outage probability is convex and the constraints are linear. Therefore, we can solve this as a convex optimization problem as illustrated in the following key result.

Proposition 3: The optimal power allocation that minimizes the outage probability of the considered underlay cognitive system with AF multihop relaying and TAS/MRC per hop with proportional interference constraint is given by

Proof: It follows the proof of Corollary 1. 


$$
\begin{aligned}
\Phi_{k, 1}= & \left(\frac{1}{\bar{\gamma}_{k, \mathrm{I}}}\right)^{L_{T}}\left(\frac{1}{\Gamma\left(N_{R}+1\right)}\right)^{N_{S}}\left(\frac{1}{\Omega_{k, h}}\right)^{N_{S} N_{R}} U\left(L, N_{S} N_{R}+L_{T}+1, \frac{1}{\bar{\gamma}_{k, \mathrm{I}}}\right) \mu^{-N_{S} N_{R}} F_{\lambda_{k, g}}\left(\frac{Q_{I}}{Q_{P}}\right), \\
\Phi_{k, 2} & =\left(\frac{1}{\bar{\gamma}_{k, \mathrm{I}}}\right)^{L_{T}}\left(\frac{1}{\Gamma\left(N_{R}+1\right)}\right)^{N_{S}}\left(\frac{1}{\Omega_{k, h}}\right)^{N_{S} N_{R}} U\left(L, N_{S} N_{R}+L_{T}+1, \frac{1}{\bar{\gamma}_{k, \mathrm{I}}}\right) \\
& \times \frac{L_{T}}{\Gamma(N)}\left(\Omega_{k, g}\right)^{N_{S} N_{R}} \sum_{k_{3}=0}^{L_{T}-1}\left(\begin{array}{c}
L_{T}-1 \\
k_{3}
\end{array}\right)(-1)^{k_{3}} \Gamma\left(k_{3}+1\right) \sum_{\left|\mathbf{c}_{\mathbf{1}}\right|=k_{3}} \mu_{l}\left(k_{3}+1\right)^{-t_{1}} \Gamma\left(t_{1}, \frac{Q_{I}\left(k_{3}+1\right)}{Q_{P} \Omega_{k, g}}\right) .
\end{aligned}
$$

$$
P_{k}^{*}=\left\{\begin{array}{l}
\frac{\left(N_{S} N_{R} \Psi_{k}\left(\gamma_{\mathrm{th}}\right)^{N_{S} N_{R}}\right)^{1+N_{S} N_{R}}}{\sum_{k=1}^{K}\left(N_{S} N_{R} \Psi_{k} \gamma_{\mathrm{th}^{N} \mathrm{~N}_{\mathrm{R}}}\right)^{\frac{1}{N_{S} N_{R}}}} P_{\mathrm{tot}} ; P_{\mathrm{tot}} \leq \sum_{k=1}^{K} \frac{I_{P}}{\lambda_{k, g}} \\
\frac{\left(\frac{N_{S} N_{R}}{\lambda_{k, g}} \Psi_{k}\left(\gamma_{\mathrm{th}}\right)^{N_{S} N_{R}}\right)^{1+N_{S} N_{R}} I_{P}}{\sum_{k=1}^{K}\left(\frac{N_{S} N_{R} \Psi_{k}}{\left(\lambda_{k, g} \gamma_{\mathrm{th}}\right)^{-N_{S} N_{R}}}\right)^{\frac{1}{N_{S} N_{R}}}} ; P_{\mathrm{tot}}>\sum_{k=1}^{K} \frac{I_{P}}{\lambda_{k, g}} .
\end{array}\right.
$$

Proof: See Appendix D.

\section{END-TO-END SINR WITH INTERFERENCE CANCELlation}

This section discusses the underlay cognitive spectrum with AF multihop relaying and using the combination of TAS and receive IC in each relaying hop. Specifically, it is considered that the receive antennas $N_{R}$ per hop are not sufficiently spaced to realize diversity gain, and hence, they are used for receive IC. On the other hand, transmit diversity per hop is exploited using TAS. When SU receive antennas are assumed to be closely separated (high correlation), and based on the estimates of DoAs of different received signal components, the SU receive antenna array radiation pattern per hop can be configured in order to reduce the effect of interference from PU transmit stations through a null-starring technique. To this end, the received signal at relay $S_{k}$ can be expressed

$$
\mathbf{y}_{k}=\sqrt{P_{k-1} \Omega_{k, h}} h_{k} x_{k-1} \mathbf{s}_{k}+\sqrt{P_{T} \Omega_{k, f}} \sum_{l=1}^{L} f_{k l q} \hat{x} \mathbf{s}_{k l q}+\mathbf{n}_{k},
$$

where $\mathbf{s}_{k}$ is a $1 \times N_{R}$ steering vector in the direction of the desired signal at the $S_{k}, \mathbf{s}_{k l q}$ is a $1 \times N_{R}$ steering vector in the direction of the $l$ th PU interference at $S_{k}$, $\left|h_{k}\right|=\max _{1 \leq i \leq N_{S}}\left|h_{k i}\right|$ (following the TAS processing). The received signal $\mathbf{y}_{k}$ is weighted by the array processing vector $\mathbf{w}_{k}$ to process interfering signals according to the adopted receive IC algorithm, with the objective to provide a maximum normalized gain of unity in the direction of the desired signal, which results in $\left|\mathbf{s}_{k} \mathbf{w}_{k}^{H}\right|=1$. Following the same treatment in section III, the end-to-end SINR at relay $S_{k}$ can be expressed as

$$
\gamma_{k}=\min \left(\mathcal{Q}_{P}, \frac{\mathcal{Q}_{I}}{\lambda_{k, g}}\right) \frac{\lambda_{k, h}}{1+\gamma_{k, \mathrm{I}, \mathrm{tot}}}
$$

where $\lambda_{k, h}=\max _{1 \leq i \leq N_{S}}\left|h_{k i}\right|^{2}$ and $\gamma_{k, \mathrm{I}, \text { tot }}=$ $\sum_{l=1}^{L} \bar{\gamma}_{k, \mathrm{I}}\left|f_{k l q}\right|^{2}\left|\mathbf{s}_{k l q} \mathbf{w}_{k}^{H}\right|^{2}$ represents the residual INR, wherein $\left|\mathbf{s}_{k l q} \mathbf{w}_{k}^{H}\right|^{2}$ implements two different IC algorithms, which are described below. The distributions of $\lambda_{k, h}$ are given by (8) and (6) but with replacing $N_{R}=1$. Moreover, the distribution of $\lambda_{k, g}$ remains the same as (8) and (6). The following subsections describe the two IC algorithms under consideration. More details can be found in [31].

\section{A. Dominant IC Algorithm}

This dominant receive IC, denoted previously as DRIC, algorithm requires ordering interference signal powers according to their levels, and attempts to eliminate the most dominant set of them through adjusting the weight vector $\mathbf{w}_{k}$. Therefore, the effect of imprecise ordering of interference powers can have significant impact on the expected outcomes.

Define $\zeta_{k, l, \mathrm{I}}=f_{k l q} \mathbf{s}_{k l q} \mathbf{w}_{k}^{H}$, for $l=1,2, \ldots, L$. Then let $\left|\zeta_{k,(1), \mathrm{I}}\right|^{2}<\left|\zeta_{k,(2), \mathrm{I}}\right|^{2}<\ldots<\left|\zeta_{k,(L), \mathrm{I}}\right|^{2}$ as the order statistics obtained by arranging $\left\{\left|\zeta_{k, l, \mathrm{I}}\right|^{2}\right\}_{l=1}^{L}$ in an increasing order of magnitude. Assuming that the receive station at relay $S_{k}$ attempts to eliminate the most effective $N_{R}-1$ (maximum number of interference sources that can be nulled) interference powers using the full-size receive antenna array, the total residual INR becomes $\gamma_{k, \mathrm{I} \text {,tot }}=\bar{\gamma}_{k, \mathrm{I}} \sum_{l=1}^{L_{A}}\left|\zeta_{k,(l), \mathrm{I}}\right|^{2}$, where $L_{A}=L_{T}-\left(N_{R}-1\right)$. Note that, when receive array is overloaded (i.e., $L_{T}>N_{R}-1$ ), $L_{A}>0$ and hence there will be residual impact of interference. On the other hand, when $L_{T} \leq N_{R}-1, L_{A}=0$ and relay $S_{k}$ can be interference-free. Therefore, $L_{A}$ is bounded as $0 \leq L_{A} \leq L_{T}$.

Considering the impact of outdated order statistics of $\left\{\left|\zeta_{k, l, \mathrm{I}}\right|^{2}\right\}_{l=1}^{L}$, which is reflected through the parameter $\tau$ being the excess time delay, and according to [24], and [27], the distribution of $\gamma_{k, \text { I,tot }}$ can be expressed as

$$
\begin{aligned}
& f_{\gamma_{k, \mathrm{I}, \mathrm{tot} \mid 0 \leq \tau<+\infty}}(x)=\frac{1}{\bar{\gamma}_{k, \mathrm{I}}}\left(\begin{array}{c}
L_{T} \\
L_{A}
\end{array}\right) \sum_{g=0}^{L_{A}-1}(-1)^{g}\left(\begin{array}{c}
L_{A} \\
g
\end{array}\right) \beta_{1}\left(\rho_{k, \mathrm{I}}\right) \\
& \times\left(\frac{L_{A}-g}{N_{R}-1}\right)^{L_{A}-1} \exp \left(\frac{-x\left(\beta_{2}\left(\rho_{\mathrm{I}}\right) \cdot\left(L_{T}-g\right)\right.}{\left(\bar{\gamma}_{k, \mathrm{I}}\left(L_{A}-g\right)\right)}\right) ; x \geq 0,
\end{aligned}
$$

where $\beta_{1}\left(\rho_{\mathrm{I}}\right) \triangleq\left(\rho_{\mathrm{I}}^{2}\right)^{1-L_{A}}\left(1+\frac{\left(1-\rho_{\mathrm{I}}^{2}\right)\left(N_{R}-1\right)}{L_{A}-g}\right)^{L_{A}-2}, \beta_{2}\left(\rho_{\mathrm{I}}\right) \triangleq$ $\left(1+\frac{\left(1-\rho_{\mathrm{I}}^{2}\right)\left(N_{R}-1\right)}{L_{A}-g}\right)^{-1}$, and Clark's model suggests that $\rho_{\mathrm{I}}=$ $\left|J_{0}\left(2 \pi \Delta f_{m} \tau\right)\right|$, where $J_{0}(x)$ is the Bessel function of the first kind with zero-order, and $\Delta f_{m}$ is the maximum Doppler spread, and $\tau$ is the feedback delay. Note that the best interference cancellation happens when $\tau=0$, whereas the 
order statistics of interference powers becomes useless when $\tau \rightarrow+\infty$.

\section{B. Adaptive IC Algorithm}

The adaptive receive IC, denoted previously as ARIC, algorithm does not require ordering the interference power levels, and therefore, it is relatively less complex than the previous algorithm. In addition, this ordering may return marginal benefits, particularly when these estimates are outdated or when the number of strong interferers is relatively large. Herein, the adaptive IC adjusts the number of used receive antennas for IC according to the experienced level of interference. Specifically, the possible occasions of low interference effect can be captured and reflected into lowering the receive processing.

The scheme aims to reduce the number of active receive antennas by adaptively activating as many antennas as necessary to have the total residual INR below a specific threshold, $\gamma_{\mathrm{I}, T}$. According to [28], distribution of the total residual INR $\gamma_{k, \mathrm{I}, \mathrm{tot}}$ in this case can be expressed as

$$
\begin{aligned}
f_{\gamma_{k, \mathrm{I}, \mathrm{tot}}}(x) & =\frac{1}{\Gamma\left(L_{A}\right)}\left(\frac{1}{\bar{\gamma}_{k, \mathrm{I}}}\right)^{L_{A}} x^{L_{A}-1} e^{-x / \bar{\gamma}_{k, \mathrm{I}} \mathrm{U}}\left(x-\gamma_{\mathrm{I}, T}\right) \\
& +\left(\frac{1}{\Gamma\left(L_{T}\right)}\left(\frac{1}{\bar{\gamma}_{k, \mathrm{I}}}\right)^{L_{T}} x^{L_{T}-1} e^{-x / \bar{\gamma}_{k, \mathrm{I}}}+e^{-\gamma_{\mathrm{I}, T} / \bar{\gamma}_{k, \mathrm{I}}}\right. \\
& \left.\times \sum_{h=2}^{L_{R}} \frac{\left(\frac{1}{\bar{\gamma}_{k, \mathrm{I}}}\right)^{\iota}}{\Gamma(\iota)} x^{\iota-1}\right)\left(\mathrm{U}(x)-\mathrm{U}\left(x-\gamma_{\mathrm{I}, T}\right)\right),
\end{aligned}
$$

where $\iota=L_{T}-(h-1)$. Note that when all receive antennas are active, it implies that $\gamma_{k, \mathrm{I} \text {,tot }}$ is greater than $\gamma_{\mathrm{I}, T}$, and hence, its distribution $\gamma_{\mathrm{I} \text {,tot }}$ becomes similar to that in (5). On the other hand, when $\gamma_{k, \mathrm{I} \text {,tot }}<\gamma_{\mathrm{I}, T}$, the active number of receive antennas becomes variable. Therefore, the distribution $\gamma_{k, \mathrm{I}, \text { tot }}$ is for the effect of the interference powers distributions and the randomness in the number of active receive antennas. The interference-free case with $\gamma_{k, \text { I,tot }}=0$ in (24)-(25) can be reached only if $L_{T} \leq N_{R}-1$, and $\gamma_{\mathrm{I}, T} \rightarrow 0$ in (25), at which $L_{A}=0$.

\section{End-TO-End OUTAGE PROBABILITY With INTERFERENCE CANCELLATION}

This section investigates the outage probability of underlay cognitive MIMO with AF mutlihop relaying, and employing TAS/DRIC and TAS/ARIC approaches in the presence of multiple PU interference per hop. Specifically, we obtain new analytical results for the exact and asymptotic end-to-end outage probability of these considered systems.

\section{A. Results With TAS/DRIC}

1) Outage Probability: We present a new closed-form outage probability formula for underlay cognitive system with AF multihop relaying and TAS/DRIC per hop in the following result.

Theorem 2: The upper bounded end-to-end SINR $\gamma_{\mathrm{E} 2 \mathrm{E}}$ outage probability of underlay cognitive system with AF multihop relaying and TAS/DRIC per hop is expressed by (9), where $F_{\gamma_{k}}\left(\gamma_{\mathrm{th}}\right)$ in this case can be derived with the results shown in (26) on the top of the next page, where $a_{1}=\lambda_{2}-k$, $\alpha=\left(\frac{\beta_{2}\left(\rho_{I}\left(L_{T}-g\right)\right)}{\bar{\gamma}_{k, \mathrm{I}}\left(L_{T}-g\right)}+\frac{\mathcal{Q}_{I} k_{1}}{\mathcal{Q}_{P}}\right)$ and $W_{a, b}($.$) is the Whittaker$ function [33, 9.220.4].

Proof: It follows footsteps of analysis in Appendix A.

Note that Theorem 2 involves standard functions and only a special function, which can be efficiently computed in standard mathematical software packages.

2) Asymptotic Analysis: In this subsection, we derive the asymptotic outage probability results considering both the proportional and the fixed interference constraint scenarios with the use of TAS/DRIC scheme per hop.

a) Proportional interference power constraint: The following key result gives the asymptotic outage probability of the treated case herein.

Proposition 4: The asymptotic outage probability of underlay cognitive system with AF multihop relaying and TAS/DRIC per hop, under the condition that $\mathcal{Q}_{I}=\mu \mathcal{Q}_{P}$ as $\mathcal{Q}_{P} \rightarrow \infty$, is given by

$$
P_{\mathrm{out}}^{\infty}\left(\gamma_{\mathrm{th}}\right) \approx \sum_{k=1}^{K} \Xi_{k}\left(\frac{\gamma_{\mathrm{th}}}{\mathcal{Q}_{P}}\right)^{N_{S}}
$$

where $\Xi_{k}$ is given in (28) on the next page.

Proof: By utilizing series expansion of the exponential function and neglecting the higher order terms, it follows that $F_{\lambda_{k, h}}(x) \approx\left(x / \Omega_{k, h}\right)^{N_{S}}$, and then repeating the same procedure adopted in Appendix B, the final result can be obtained.

Based on Proposition 4, we present the following Corollaries.

Corollary 4: The diversity and coding gains of underlay cognitive system with AF multihop relaying and TAS/DRIC per hop, under the condition that $\mathcal{Q}_{I}=\mu \mathcal{Q}_{P}$ as $\mathcal{Q}_{P} \rightarrow \infty$, are given respectively by $\mathcal{G}_{D}=N_{S}$ and

$$
\mathcal{G}_{C}=\min _{k=1 \ldots K} \frac{\left(\Xi_{k}\right)^{-\frac{1}{N_{S}}}}{\gamma_{\mathrm{th}}}
$$

Proof: It follows proof Corollary 1.

Corollary 5: The DMT of underlay cognitive system with AF multihop relaying and TAS/DRIC per hop, under the condition that $\mathcal{Q}_{I}=\mu \mathcal{Q}_{P}$ as $\mathcal{Q}_{P} \rightarrow \infty$, is given by $d(\hat{r})=(1-2 \hat{r}) N_{S}$.

Proof: It follows the same footsteps in Appendix C.

b) Fixed interference power constraint: For such, we present the following key results.

Proposition 5: The asymptotic outage probability of underlay cognitive system with AF multihop relaying and TAS/ARIC per hop, under the condition that $\mathcal{Q}_{I}$ is fixed as $\mathcal{Q}_{P} \rightarrow \infty$, is given by

$$
P_{\mathrm{out}}^{\infty}\left(\gamma_{\mathrm{th}}\right) \approx \sum_{k=1}^{K}\left(\Delta_{k, 1}\left(\frac{1}{\mathcal{Q}_{P}}\right)^{N_{S}}+\Delta_{k, 2}\left(\frac{1}{\mathcal{Q}_{I}}\right)^{N_{S}}\right) \gamma_{\mathrm{th}}^{N_{S}},
$$

where $\Delta_{k, 1}$ and $\Delta_{k, 2}$ are given respectively by (31) and (32).

Proof: It follows similar proof of Proposition 4. 


$$
\begin{aligned}
& F_{\gamma_{k}}\left(\gamma_{\mathrm{th}}\right)=F_{\lambda_{k, g}}\left(\frac{\mathcal{Q}_{I}}{\mathcal{Q}_{P}}\right)\left(1-\frac{1}{\bar{\gamma}_{k, \mathrm{I}}}\left(\begin{array}{c}
L_{T} \\
L_{A}
\end{array}\right) \sum_{g=0}^{L_{A}-1}\left(\begin{array}{c}
L_{A} \\
g
\end{array}\right)(-1)^{g} \beta_{1}\left(\rho_{I}\right)\left(\frac{L_{A}-g}{L_{T}-L_{A}}\right)^{L_{A}-1} \sum_{k_{1}=0}^{N_{S}}\left(\begin{array}{c}
N_{S} \\
k_{1}
\end{array}\right)(-1)^{k_{1}}\right. \\
& \left.\quad \times \exp \left(\frac{-k_{1} \gamma_{\mathrm{th}}}{\Omega_{k, h} \mathcal{Q}_{P}}\right)\left(\frac{\beta_{2}\left(\rho_{I}\right)\left(L_{T}-g\right)}{\bar{\gamma}_{k, \mathrm{I}}\left(L_{A}-g\right)}+\frac{k_{1} \gamma_{\mathrm{th}}}{\Omega_{k, h} \mathcal{Q}_{P}}\right)^{-1}\right)+\left(1-F_{\lambda_{k, g}}\left(\frac{\mathcal{Q}_{I}}{\mathcal{Q}_{P}}\right)\right) \\
& \quad-\frac{L_{T}}{\Gamma(N) \bar{\gamma}_{k, g}} \sum_{k_{1}=0}^{N_{S}}\left(\begin{array}{c}
N_{S} \\
k_{1}
\end{array}\right)(-1)^{k_{1}} \sum_{k_{3}=0}^{L_{T}-1}\left(\begin{array}{c}
L-1 \\
k_{3}
\end{array}\right)(-1)^{k_{3}} \Gamma\left(k_{3}+1\right) \sum_{\left|\mathbf{c}_{1}\right|=k_{3}} \mu_{l}\left(\frac{1}{\Omega_{k, g}}\right)^{\lambda_{l}+N-1} \\
& \quad \times \exp \left(-\frac{\mathcal{Q}_{I}}{Q_{P}} \phi\right) \sum_{k=0}^{\mu_{2}-1} \frac{\Gamma\left(\mu_{2}\right)}{\Gamma(k+1)}\left(\frac{\mathcal{Q}_{I}}{\mathcal{Q}_{P}}\right)^{k}\left(\frac{1}{\phi}\right)^{a_{1}} \frac{1}{\bar{\gamma}_{k, \mathrm{I}}}\left(\begin{array}{c}
L_{T} \\
L_{A}
\end{array}\right) \sum_{g=0}^{L_{A}-1}\left(\begin{array}{c}
L_{A} \\
g
\end{array}\right)(-1)^{g} \beta_{1}\left(\rho_{I}\right) \\
& \quad \times\left(\frac{L_{A}-g}{L_{T}-L_{A}}\right)^{L_{A}-1} \frac{1}{\alpha}\left(\frac{\alpha \phi \mathcal{Q}_{I} \Omega_{k, h}}{k_{1} \gamma_{\mathrm{th}}}\right)^{\frac{a_{1}}{2}} \exp \left(\frac{\alpha \phi \mathcal{Q}_{I} \Omega_{k, h}}{2 k_{1} \gamma_{\mathrm{th}}}\right) W_{-\frac{a_{1}}{2}, \frac{1-a_{1}}{2}}\left(\frac{\alpha \phi \mathcal{Q}_{I} \Omega_{k, h}}{k_{1} \gamma_{\mathrm{th}}}\right)
\end{aligned}
$$

$$
\begin{aligned}
\Xi_{k} & =\frac{1}{\bar{\gamma}_{k, \mathrm{I}}}\left(\begin{array}{c}
L_{T} \\
L_{A}
\end{array}\right) \sum_{g=0}^{L_{A}-1}(-1)^{g}\left(\begin{array}{c}
L_{A} \\
g
\end{array}\right) \beta_{1}\left(\rho_{\mathrm{I}}\right)\left(\frac{L_{A}-g}{N_{R}-1}\right)^{L_{A}-1} \exp \left(\frac{\beta_{2}\left(\rho_{\mathrm{I}}\right) \cdot(L-g)}{\bar{\gamma}_{k, \mathrm{I}}\left(L_{A}-g\right)}\right) \\
& \times\left(\frac{\beta_{2}\left(\rho_{\mathrm{I}}\right) \cdot\left(L_{T}-g\right)}{\bar{\gamma}_{k, \mathrm{I}}\left(L_{A}-g\right)}\right)^{-\left(N_{S}+1\right)} \Gamma\left(N_{S}+1, \frac{\beta_{2}\left(\rho_{\mathrm{I}}\right) \cdot\left(L_{T}-g\right)}{\bar{\gamma}_{k, \mathrm{I}}\left(L_{A}-g\right)}\right)\left(F_{\lambda_{k, g}}(\mu)+\frac{N_{S}}{\Gamma(N)} \mu^{-N_{S}}\right. \\
& \left.\times \sum_{r=0}^{N_{S}-1} N_{S}-1 C_{r} \sum_{r_{p}: N} \frac{1}{\psi\left(r_{n}\right)}\left(\Omega_{k, g}\right)^{N_{S}-1}(r+1)^{-a_{3}} \Gamma\left(a_{3}, \frac{(r+1) \mathcal{Q}_{I}}{\Omega_{k, g} \mathcal{Q}_{P}}\right)\right)\left(\frac{1}{\Omega_{k, h}}\right)^{N_{S}} .
\end{aligned}
$$

$$
\begin{gathered}
\Delta_{k, 1}=\frac{1}{\bar{\gamma}_{k, \mathrm{I}}}\left(\begin{array}{c}
L_{T} \\
L_{A}
\end{array}\right) \sum_{g=0}^{L_{A}-1}(-1)^{g}\left(\begin{array}{c}
L_{A} \\
g
\end{array}\right) \beta_{1}\left(\rho_{\mathrm{I}}\right)\left(\frac{L_{A}-g}{N_{R}-1}\right)^{L_{A}-1} \exp \left(\frac{\beta_{2}\left(\rho_{\mathrm{I}}\right) \cdot\left(L_{T}-g\right)}{\bar{\gamma}_{k, \mathrm{I}}\left(L_{A}-g\right)}\right) \\
\times\left(\frac{\beta_{2}\left(\rho_{\mathrm{I}}\right) \cdot\left(L_{T}-g\right)}{\bar{\gamma}_{k, \mathrm{I}}\left(L_{A}-g\right)}\right)^{-\left(N_{S}+1\right)} \Gamma\left(N_{S}+1, \frac{\beta_{2}\left(\rho_{\mathrm{I}}\right) \cdot\left(L_{T}-g\right)}{\bar{\gamma}_{k, \mathrm{I}}\left(L_{A}-g\right)}\right) F_{\lambda_{k, g}}\left(\frac{\mathcal{Q}_{I}}{\mathcal{Q}_{P}}\right), \\
\Delta_{k, 2}=\frac{1}{\bar{\gamma}_{k, \mathrm{I}}}\left(\begin{array}{c}
L_{T} \\
L_{A}
\end{array}\right) \sum_{g=0}^{L_{A}-1}(-1)^{g}\left(\begin{array}{c}
L_{A} \\
g
\end{array}\right) \beta_{1}\left(\rho_{\mathrm{I}}\right)\left(\frac{L_{A}-g}{N_{R}-1}\right)^{L_{A}-1} \exp \left(\frac{\beta_{2}\left(\rho_{\mathrm{I}}\right) \cdot\left(L_{T}-g\right)}{\bar{\gamma}_{k, \mathrm{I}}\left(L_{A}-g\right)}\right)\left(\frac{\beta_{2}\left(\rho_{\mathrm{I}}\right) \cdot\left(L_{T}-g\right)}{\bar{\gamma}_{k, \mathrm{I}}\left(L_{A}-g\right)}\right)^{-\left(N_{S}+1\right)} \\
\times \Gamma\left(N_{S}+1, \frac{\beta_{2}\left(\rho_{\mathrm{I}}\right) \cdot\left(L_{T}-g\right)}{\bar{\gamma}_{k, \mathrm{I}}\left(L_{A}-g\right)}\right) \frac{N_{S}}{\Gamma(N)} \mu^{-N_{S}} s \sum_{r=0}^{N_{S}-1} N_{S}-1 C_{r} \sum_{r_{p}: N} \frac{1}{\psi\left(r_{n}\right)}\left(\Omega_{k, g}\right)^{N_{S}-1}(r+1)^{-a_{3}} \Gamma\left(a_{3}, \frac{(r+1) \mathcal{Q}_{I}}{\Omega_{k, g} \mathcal{Q}_{P}}\right) .
\end{gathered}
$$

Corollary 6: The diversity and coding gains of underlay cognitive system with AF multihop relaying and TAS/DRIC per hop are given respectively by $\mathcal{G}_{D}=N_{S} N_{R}$ and

$$
\mathcal{G}_{C}=\min _{k=1 \ldots K} \frac{\Delta_{k, 1}^{-\frac{1}{N_{S}}}+\left(\Delta_{k, 1} \mu\right)^{-\frac{1}{N_{S}}}}{\gamma_{\mathrm{th}}}
$$

Proof: Follow similar proof of Corollary 1.

3) Optimal Power allocation: The power allocation of the considered system with the use of TAS/DRIC per hop under proportional interference power constraint is presented in the following Proposition.

Proposition 6: The optimal power allocation that minimizes the outage probability of the considered underlay cognitive system with AF multihop relaying and TAS/DRIC per hop with proportional interference power constraint is given by

$$
P_{k}^{*}= \begin{cases}\frac{\left(N_{S} \Xi_{k}\left(\gamma_{\mathrm{th}}\right)^{N_{S}}\right)^{1+N_{S}}}{\sum_{k=0}^{K}\left(N_{S} \Xi_{k} \gamma_{\mathrm{th}^{\mathrm{N}}}\right)^{\frac{1}{N_{S}}}} P_{\mathrm{tot}} & P_{\mathrm{tot}} \leq \sum_{k=1}^{K} \frac{I_{P}}{\lambda_{k, g}} \\ \frac{\left(\frac{N_{S}}{\lambda_{k, g}} \Xi_{k}\left(\gamma_{\mathrm{th}}\right)^{N_{S}}\right)^{1+N_{S}} I_{P}}{\sum_{k=0}^{K}\left(N_{S} \Xi_{k}\left(\lambda_{k, g} \gamma_{\mathrm{th}}\right)^{N_{S}}\right)^{\frac{1}{N_{S}}}} & P_{\mathrm{tot}}>\sum_{k=1}^{K} \frac{I_{P}}{\lambda_{k, g}}\end{cases}
$$

Proof: It follows proof of Proposition 3.

\section{B. Results with TAS/ARIC}

1) Outage Probability: We present a new closed form expression for end-to-end upper bound outage probability of 
underlay cognitive system with TAS/ARIC in the following Theorem.

Theorem 3: The exact upper bound outage probability of cognitive spectrum sharing with TAS/ARIC in AF multihop relaying network is expressed by (9) where $F_{\gamma_{k}}\left(\gamma_{\mathrm{th}}\right)$ is given by (35) as shown on the top of the next page. where $\varpi=$ $\frac{Q_{I} k_{1} \theta}{Q_{P}}+\frac{1}{\bar{\gamma}_{I}}, \varpi_{2}=\frac{Q_{I} k_{1} \theta}{Q_{P}}, b_{1}, b_{2}=-\left(L_{A}-g_{1}-\mu 2+k-1\right)$, $b_{3}=-\left(L_{T}-g_{2}-\mu 2+k-1\right)$ and $b_{4}=-\left(L_{T}-h-g_{3}-\mu 2+k\right)$, $b_{5}=L-(h-1) \chi=\left(1+\gamma_{I, T} \frac{k_{1} \theta}{\phi}\right), \delta=\left(\frac{\phi}{k_{1} \theta}+\gamma_{I, T}\right)$, and $\zeta_{1}=\left(\frac{k_{1} \gamma_{t h}}{\Omega_{k, h} Q_{P}}+\frac{1}{\bar{\gamma}_{k, I}}\right)$.

Proof: See Appendix E.

2) Asymptotic Analysis: In the following we present the asymptotic analysis of Theorem 3.

a) Proportional interference power constraint: For adaptive interference cancellation, we have the following key result.

Proposition 7: The asymptotic outage probability of cognitive sharing with TAS/ARIC of AF multihop relaying network is given by

$$
P_{\text {out }}^{\infty}\left(\gamma_{\mathrm{th}}\right) \approx \sum_{k=1}^{K} \Theta_{k}\left(\frac{\gamma_{\mathrm{th}}}{\mathcal{Q}_{P}}\right)^{N_{S}}
$$

where $\Theta_{k}$ is given in (37) as shown in the top of the next page.

Proof: By repeating the same procedure adopted in Appendix A, the final result can be obtained.

Based on Proposition 7, we present the following corollary.

Corollary 7: The diversity and coding gains of cognitive sharing with TAS/ARIC of AF multihop relaying network are given respectively by $\mathcal{G}_{D}=N_{S}$ and

$$
\mathcal{G}_{C}=\min _{k=1 \ldots K} \frac{\Theta_{k}^{-\frac{1}{N_{S}}}}{\gamma_{\mathrm{th}}} .
$$

Proof: Follow similar proof of Corollary 1.

Corollary 8: The DMT of cognitive spectrum sharing with TAS/ARIC in AF multihop relaying network is given by $d(\hat{r})=$ $(1-2 \hat{r}) N_{S}$.

Proof: It follows the same footsteps in Appendix C.

b) Fixed interference power constraint: For this scenario, we present the following key result.

Proposition 8: The asymptotic outage probability of cognitive sharing with TAS/ARIC of AF multihop relaying network is given by

$$
P_{\mathrm{out}}^{\infty}\left(\gamma_{\mathrm{th}}\right) \approx \sum_{k=1}^{K}\left(\Lambda_{k, 1}\left(\frac{1}{\mathcal{Q}_{P}}\right)^{N_{S}}+\Lambda_{k, 2}\left(\frac{1}{\mathcal{Q}_{I}}\right)^{N_{S}}\right) \gamma_{\mathrm{th}}^{N_{S}}
$$

where $\Lambda_{k, 1}$ and $\Lambda_{k, 2}$ are given respectively by (40) and (41).

Proof: It follows proof of Proposition 1.

Based on Proposition 8, we present the following corollary.

Corollary 9: The diversity and coding gains of of cognitive sharing with TAS/ARIC of AF multihop relaying network are given by respectively $\mathcal{G}_{D}=N_{S}$ and

$$
\mathcal{G}_{C}=\min _{k=1 \ldots K} \frac{\left(\Lambda_{k, 1}^{-\frac{1}{N_{S}}}+\left(\Lambda_{k, 2} \mu\right)^{-\frac{1}{N_{S}}}\right)}{\gamma_{\mathrm{th}}} .
$$

Proof: Follow similar proof of Corollary 1.

3) Optimal Power allocation: The power allocation of ARIC scheme is presented in the following Proposition.

Proposition 9: The optimal power allocation that minimizes the outage probability of underlay cognitive system with multihop AF relaying and TAS/ARIC per hop is derived as

$$
P_{k}^{*}= \begin{cases}\frac{\left(N_{S} \Theta_{k}\left(\gamma_{\mathrm{th}}\right)^{N_{S}}\right)^{1+N_{S}}}{\sum_{k=0}^{K}\left(N_{S} \Theta_{k} \gamma_{\mathrm{th}} \mathrm{N}_{S}\right)^{\frac{1}{N_{S}}}} P_{\mathrm{tot}} & P_{\mathrm{tot}} \leq \sum_{k=1}^{K} \frac{I_{P}}{\lambda_{k, g}} \\ \frac{\left(\frac{N_{S}}{\lambda_{k, g}} \Theta_{k}\left(\gamma_{\mathrm{th}}\right)^{N_{S}}\right)^{1+N_{S}} I_{P}}{\sum_{k=0}^{K}\left(N_{S} \Theta_{k}\left(\lambda_{k, g} \gamma_{\mathrm{th}}\right)^{N_{S}}\right)^{\frac{1}{N_{S}}}} & P_{\mathrm{tot}}>\sum_{k=1}^{K} \frac{I_{P}}{\lambda_{k, g}}\end{cases}
$$

Proof: Follow same proof adopted in Appendix C.

According to Theorems:(1)-(3), Propositions:(1)-(9) and Corollaries:(1)-(9), we offer the following remarks to provide insights into TAS/MRC and TAS/IC schemes for underlay cognitive AF multihop system.

Remark 1: The maximum diversity outage probability gain of TAS/MRC, TAS/DRIC, and TAS/ARIC are given by $N_{S} N_{R}$, $N_{S}$, and $N_{S}$, respectively. This shows that, when the receive array antennas per hop are used for diversity, the best performance in the high SNR regime is achieved. While in low SNR (i.e. $\mathcal{Q}_{P}<10 \mathrm{~dB}$ ), the TAS/DRIC scheme provides the best performance which implies that in presence of strong interferers powers, employing receive array antennas for interference cancellation is recommended.

Remark 2: The maximum achievable gain is only feasible when the SU transmit power $\mathcal{Q}_{P}$ is directly proportional to the peak interference temperature $\mathcal{Q}_{I}$, which is desirable since it is completely independent of the primary network.

Remark 3: When $\mathcal{Q}_{P} \rightarrow \infty$, and the peak interference power $\mathcal{Q}_{I}$ is fixed, the resultant outage probability becomes saturated, and hence the achievable diversity order of the system can not be guaranteed which leads to zero diversity gain.

Remark 4: The DMTs of TAS/MRC, TAS/DRIC, and TAS/ARIC schemes are entirely dependent on the secondary network, and independent of the primary network.

Remark 5: From the results we have shown that TAS/DRIC and TAS/ARIC schemes achieve the same diversity gains which indicates that the tradeoff between the scenarios is solely characterized by their respective coding gains. Thus, we characterize the SNR gap between the two schemes as a simple ratio of their respective coding gain as

$$
\Delta_{\mathrm{Gab}}=\frac{\mathcal{G}_{C}^{\mathrm{TAS} / \mathrm{DRIC}}}{\mathcal{G}_{C}^{\mathrm{TAS} / \mathrm{ARIC}}}=\min _{k=1 \ldots K}\left(\frac{\Xi_{k}}{\Theta_{k}}\right)
$$

This indicates that, for the same outage probability, the performance of TAS/DRIC scheme outperforms the TAS/ARIC scheme by SNR gap $10 \log _{10} \Delta_{\text {Gab }}$.

\section{Simulation Results}

In this section, we present Monte Carlo simulation results to validate our analytical expressions derived in the previous sections and give a detailed investigation on the impact of the number of antennas, the number of hops, and interference outage constraints on the outage performance of cognitive mutlihop relaying systems. 


$$
\begin{aligned}
& F_{\gamma_{k}}\left(\gamma_{\mathrm{th}}\right)=\sum_{k_{1}=0}^{N_{S}}\left(\begin{array}{c}
N_{S} \\
k_{1}
\end{array}\right)(-1)^{k_{1}} \exp \left(-\frac{k_{1} \gamma_{t h}}{\Omega_{k, h} Q_{P}}\right)\left[\frac{1}{\Gamma\left(L_{A}\right)}\left(\frac{1}{\bar{\gamma}_{k, \mathrm{I}}}\right)^{L_{A}} \Gamma\left(L_{A}, \gamma_{\mathrm{I}, T} \zeta_{1}\right) \zeta_{1}^{-L_{A}}+\frac{1}{\Gamma(L)}\left(\frac{1}{\bar{\gamma}_{k, \mathrm{I}}}\right)^{L}\right. \\
& \left.\times \gamma\left(L_{T}, \gamma_{\mathrm{I}, T} \zeta_{1}\right) \zeta_{1}^{-L_{T}}+e^{-\gamma_{\mathrm{I}, \mathrm{T}} / \bar{\gamma}_{k, \mathrm{I}}} \sum_{h=2}^{N_{R}}\left(\frac{1}{\bar{\gamma}_{k, \mathrm{I}}}\right)^{L-(h-1)} \frac{1}{\Gamma\left(L_{T}-(h-1)\right)} \gamma\left(L_{T}-h+1, \gamma_{\mathrm{I}, T} \zeta_{1}\right) \zeta_{1}^{h-L_{T}-1}\right] \\
& \times \frac{L_{T}}{\Gamma(N) \Omega_{k, g}} \sum_{k_{1}=0}^{N_{S}}\left(\begin{array}{c}
N_{S} \\
k_{1}
\end{array}\right)(-1)^{k_{1}} \sum_{k_{3}=0}^{L_{T}-1}\left(\begin{array}{c}
L_{T}-1 \\
k_{3}
\end{array}\right)(-1)^{k_{3}} \Gamma\left(k_{3}+1\right) \sum_{\left|\mathbf{c}_{\mathbf{1}}\right|=k_{3}} \mu_{l}\left(\frac{1}{\Omega_{k, g}}\right)^{b_{1}} \sum_{k=0}^{\mu_{2}-1} \frac{\Gamma\left(\mu_{2}\right)}{\Gamma(k+1)}\left(\frac{Q_{I}}{Q_{P}}\right)^{k} \\
& \times \exp \left(-\frac{Q_{I}}{Q_{P}} \phi\right)\left(\frac{1}{\phi}\right)^{\mu_{2}-k} \times\left[\frac{1}{\Gamma\left(L_{A}\right)}\left(\frac{1}{\bar{\gamma}_{k, \mathrm{I}}}\right)^{L_{A}} \sum_{g_{1}=0}^{L_{A}-1}\left(\begin{array}{c}
L_{A}-1 \\
g_{1}
\end{array}\right)(-1)^{g_{1}} \varpi^{\frac{b_{2}}{2}-1}\left(\frac{\phi}{k_{1} \theta}\right)^{L_{A}+\frac{b_{2}}{2}-1}\right. \\
& \times \chi^{-\frac{b_{2}}{2}} \exp \left(\frac{-\varpi}{2} \delta\right) W_{-\frac{b_{2}}{2}, \frac{1-b_{2}}{2}}(\varpi \delta)+\frac{1}{\Gamma\left(L_{T}\right)}\left(\frac{1}{\bar{\gamma}_{k, \mathrm{I}}}\right)^{L_{T}} \sum_{g_{2}=0}^{L_{T}-1}\left(\begin{array}{c}
L_{T}-1 \\
g_{2}
\end{array}\right)(-1)^{g_{2}} \varpi^{\frac{a_{3}}{2}-1}\left(\frac{\phi}{k_{1} \theta}\right)^{L_{T}+\frac{b_{3}}{2}-1} \\
& \times \exp \left(\frac{\varpi \phi}{2 k_{1} \theta}\right)\left(W_{-\frac{b_{3}}{2}, \frac{1-b_{3}}{2}}\left(\frac{\varpi \phi}{k_{1} \theta}\right)-\chi^{-\frac{b_{3}}{2}} \exp \left(-\frac{\varpi \gamma_{I, T}}{2}\right) W_{-\frac{b_{3}}{2}, \frac{1-b_{3}}{2}}(\zeta \delta)\right)+\sum_{h=2}^{N_{R}} \frac{\exp -\frac{\gamma_{1, T}}{\bar{\gamma}_{k, \mathrm{I}}}\left(\frac{1}{\bar{\gamma}_{k, 1}}\right)^{b_{5}}}{\Gamma\left(b_{5}\right)} \\
& \left.\times \sum_{g_{3}=0}^{L_{T}-h}\left(\begin{array}{c}
L_{T}-h \\
g_{3}
\end{array}\right)(-1)^{g_{3}} \frac{\exp \left(\frac{\varpi_{2} \phi}{2 k_{1} \theta}\right)}{\varpi_{2}^{-\frac{b_{4}}{2}+1}\left(\frac{\phi}{k_{1} \theta}\right)^{-L_{T}+h-\frac{b_{4}}{2}}}\left(W_{-\frac{a_{4}}{2}, \frac{1-a_{4}}{2}}\left(\frac{\varpi_{2} \phi}{k_{1} \theta}\right)-\frac{\exp \left(-\frac{\varpi_{2} \gamma_{I, T}}{2}\right)}{\chi^{\frac{a_{4}}{2}}} W_{-\frac{b_{4}}{2}, \frac{1-b_{4}}{2}}\left(\varpi_{2} \delta\right)\right)\right],
\end{aligned}
$$

$$
\begin{array}{r}
\Theta_{k}=\sum_{j=0}^{N_{S}}\left(\begin{array}{c}
N_{S} \\
j
\end{array}\right)\left[\frac{\bar{\gamma}_{k, \mathrm{I}}^{j}}{\Gamma\left(L_{A}\right)} \Gamma\left(L_{A}+j, \frac{\gamma_{\mathrm{I}, T}}{\bar{\gamma}_{I}}\right)+\frac{\bar{\gamma}_{I}^{j}}{\Gamma\left(L_{T}\right)} \gamma\left(L_{T}+j, \frac{\gamma_{\mathrm{I}, T}}{\bar{\gamma}_{k, \mathrm{I}}}\right)+e^{-\gamma_{\mathrm{I}, T} / \bar{\gamma}_{k, \mathrm{I}}} \sum_{h=2}^{N_{R}}\left(\frac{1}{\bar{\gamma}_{k, \mathrm{I}}}\right)^{b_{5}} \frac{\gamma_{\mathrm{I}, T}^{b_{6}}}{\left(b_{6}\right) \Gamma\left(b_{5}\right)}\right] \\
\left(Q_{P}^{N_{S}} F_{\gamma_{k, g}}(\mu)+\left(\frac{\Omega_{k, g}}{Q_{I}}\right)^{N_{S}} \frac{L_{T}}{\Gamma(N)} \sum_{k_{3}=0}^{L_{T}-1}\left(\begin{array}{c}
L_{T}-1 \\
k_{3}
\end{array}\right)(-1)^{k_{3}} \Gamma\left(k_{3}+1\right) \sum_{\left|\mathbf{c}_{1}\right|=k_{3}} \mu_{l} \frac{\Gamma\left(N_{S}+\lambda_{l}+N, \frac{Q_{I}\left(k_{3}+1\right)}{Q_{P} \Omega_{k, g}}\right)}{\left(k_{3}+1\right)^{\left(N_{S}+\lambda_{l}+N\right)}}\right) .
\end{array}
$$

$$
\begin{gathered}
\Lambda_{k, 1}=\sum_{j=0}^{N_{S}}\left(\begin{array}{c}
N_{S} \\
j
\end{array}\right) F_{\gamma_{k, g}}(\mu)\left[\frac{\bar{\gamma}_{k, \mathrm{I}}^{j}}{\Gamma\left(L_{A}\right)} \Gamma\left(L_{A}+j, \frac{\gamma_{\mathrm{I}, T}}{\bar{\gamma}_{I}}\right)+\frac{\bar{\gamma}_{I}^{j}}{\Gamma(L)} \gamma\left(L+j, \frac{\gamma_{\mathrm{I}, T}}{\bar{\gamma}_{k, \mathrm{I}}}\right)+e^{-\gamma_{\mathrm{I}, T} / \bar{\gamma}_{k, \mathrm{I}}} \sum_{h=2}^{N_{R}}\left(\frac{1}{\bar{\gamma}_{k, \mathrm{I}}}\right)^{b_{5}} \frac{\gamma_{\mathrm{I}, T}^{b_{6}}}{\left(b_{6}\right) \Gamma\left(b_{5}\right)}\right] \\
\Lambda_{k, 2}=\sum_{j=0}^{N_{S}}\left(\begin{array}{c}
N_{S} \\
j
\end{array}\right)\left[\frac{\bar{\gamma}_{k, \mathrm{I}}^{j}}{\Gamma\left(L_{A}\right)} \Gamma\left(L_{A}+j, \frac{\gamma_{\mathrm{I}, T}}{\bar{\gamma}_{I}}\right)+\frac{\bar{\gamma}_{I}^{j}}{\Gamma\left(L_{T}\right)} \gamma\left(L+j, \frac{\gamma_{\mathrm{I}, T}}{\bar{\gamma}_{k, \mathrm{I}}}\right)+e^{-\gamma_{\mathrm{I}, T} / \bar{\gamma}_{k, \mathrm{I}}} \sum_{h=2}^{N_{R}}\left(\frac{1}{\bar{\gamma}_{k, \mathrm{I}}}\right)^{b_{5}} \frac{\gamma_{\mathrm{I}, T}^{b_{6}}}{\left(b_{6}\right) \Gamma\left(b_{5}\right)}\right] \\
\Omega_{k, g}^{N_{S}} \frac{L_{T}}{\Gamma(N)} \sum_{k_{3}=0}^{L_{T}-1}\left(\begin{array}{c}
L-1 \\
k_{3}
\end{array}\right)(-1)^{k_{3}} \Gamma\left(k_{3}+1\right) \sum_{\left|\mathbf{c}_{\mathrm{I}}\right|=k_{3}} \mu_{l} \frac{\Gamma\left(N_{S}+\lambda_{l}+N, \frac{Q_{I}\left(k_{3}+1\right)}{Q_{P} \Omega_{k, g}}\right)}{\left(k_{3}+1\right)^{\left(N_{S}+\lambda_{l}+N\right)}}
\end{gathered}
$$

Fig. 2 plots the outage probability of TAS/MRC scheme for different transmit antenna $N_{S}=\{1,2,3\}$ and outage threshold $\gamma_{\text {th }}=\{-5,2\} \mathrm{dB}$ when $\mathcal{Q}_{P}=\mu \mathcal{Q}_{I}$. For $\mathrm{SU}$ network, we assume a co-linear network where all hops are located on a straight line. In particular, we assume that the cognitive multihop network takes place over $K=3$. For the PU network, we assume the primary transmitters and receivers are set to $L=5$ and $N=3$, respectively. In addition, the interference from the primary transmitters is set as $\bar{\gamma}_{k, \mathrm{I}}=3$ dB. As shown in the figure, when $N_{S}$ increases from $1 \rightarrow 3$, the outage probability decreases. This is quite expected since increasing $N_{S}$ always translates into a higher diversity order. Most importantly, it can be observed that the analytical results are in exact agreement with Monte-Carlo simulation results, and asymptotic results are sufficiently tight in the high SNR. The same figure shows the impact of outage threshold on the outage performance. As expected, increasing the outage threshold decreases the outage performance.

Fig. 3 compares the outage performance of the proposed TAS/MRC, TAS/DRIC, and TAS/ARIC schemes of cognitive 
multihop relaying system when $\mathcal{Q}_{P}=\mathcal{Q}_{I}$, and $N_{R}=$ $\{3,4\}$. As can be readily observed, for all three scenarios, the proposed TAS/MRC scheme outperforms TAS/DRIC and TAS/ARIC in the high SNR regime. Interestingly, in the low SNR regime, TAS/MRC and TAS/ARIC achieve similar outage performance while TAS/DRIC performs the best. This shows that TAS/DRIC and TAS/ARIC schemes can be good candidates for cancelling the effects of the dominant interfering powers and hence improve the end-to-end performance in the low SNR. It can be further noticed that TAS/DRIC outperforms TAS/ARIC scheme but the later has low processing complexity and power consumption which is achieved by adaptively activates receive antennas to have the residual total instantaneous power within a tolerable range $\gamma_{k, T}$.

In Fig. 4, we consider the scenario where $\mathcal{Q}_{P}$ is independent of $\mathcal{Q}_{I}$ to see its impact on the outage performance of the proposed TAS/MRC, TAS/DRIC, and TAS/ARIC schemes when $K=3, \gamma_{\mathrm{th}}=0 \mathrm{~dB}$, and $\mathcal{Q}_{I}=20 \mathrm{~dB}$. From the shown figure, it can be observed that the outage probability of the considered system becomes saturated as a result of the fixed interference temperature constraint. It is important to observe that the outage error floor and outage performance can be improved by adopting more transmits antenna per hop to achieve high diversity gain, and more receive antennas to cancel most of dominant dominant interfering powers. As it can be noticed that the outage performance of TAS/IC schemes under fixed interference power constraint $\mathcal{Q}_{I}$ is more affected compared to the TAS/MRC scheme in high SNR.

Next, in Fig. 5 and 6, we consider the effect of increasing number PU transmitters and hops, respectively when $\mathcal{Q}_{P}=$ $\mathcal{Q}_{I}, \gamma_{\mathrm{th}}=0, \mathrm{~dB}$, and $\bar{\gamma}_{k, \mathrm{I}}=3 \mathrm{~dB}$ on the outage performance. We can notice from the previous analysis, and based on the results in corollaries that the diversity gains for all schemes are independent of the number of hops and PU transmitters, however, they only affect the coding gains by shifting the curves. Therefore, from the obtained results in both figures, we notice that increasing the number of hops $1 \rightarrow 3$ and number of PU transmitters $2 \rightarrow 4$ decreases the outage performance by shifting the curves to the right while the slops of the curves remain the same.

Fig. 7 shows the outage performance of the proposed TAS/DRIC scheme with different system set-up when $\mathcal{Q}_{I}=$ $\mathcal{Q}_{P}$. It is observed that the analytical results are in exact agreement with Monte-Carlo simulation results, and asymptotic results are sufficiently tight in the high SNR. Since the receive antennas $N_{R}$ is employed to provide interference cancellation, it can be observed when the receive antennas increases, more dominant interferers are cancelled, and hence system performance improvement appears. In addition, the results illustrate the impact of $\rho_{I}$ on the outage performance i.e., when $\rho_{I}=1$, it corresponds to perfect interference reduction/cancellation, and when $\rho_{I}=0$, it indicates that the interference cancellation of the interfering signals is made randomly. In particular, it can be shown that due the imperfect ranking of interferers signals which is quantified through the reduction of the value of $\rho_{I}$, the outage performance deteriorates, and the positive impact of interference cancellation on the outage performance reduces. A close look at the outage performance reduction, we observe

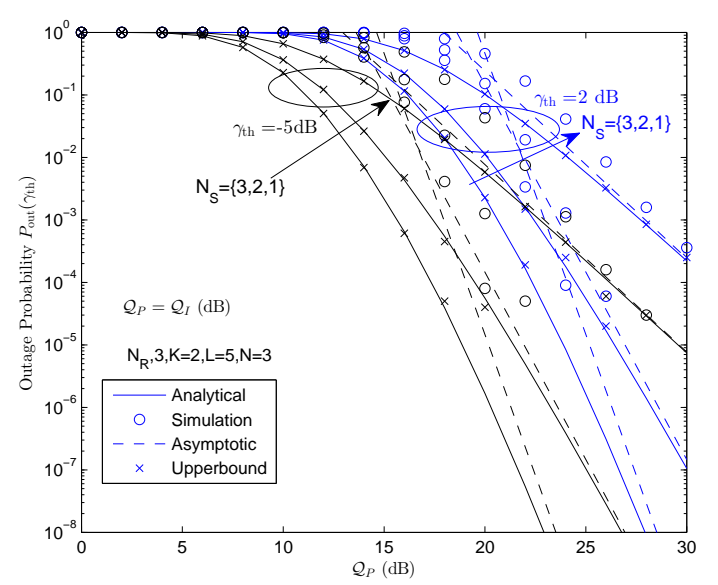

Fig. 2. The outage probability of cognitive MIMO multihop in spectrum sharing with TAS/MRC when $\mathcal{Q}_{P}=\mathcal{Q}_{I}$.

that the gap difference between $\rho_{I}=1$ and $\rho_{I}=0.5$ is almost $3 \mathrm{~dB}$, this indicates how much the performance is degraded due to imperfect ranking of the interference caused by the PU transmitters.

Fig. 8 plots the exact of and asymptotic outage probability of the secondary network with ARIC. From the shown figure, it can be observed that the outage probability of the considered system becomes saturated as a result of the fixed interference temperature constraint. It is important to observe that the outage error floor and outage performance can be improved by adopting more transmit antennas per hop to achieve high diversity gain, and more receive antennas to cancel more interference. It can be shown that increasing number of hops and the number of PUs receivers degrade the end-to-end performance.

Fig. 9 depicts the outage probability of the secondary network with ARIC versus $\gamma_{\mathrm{I}, T}$ for different SU $N_{R}$ receive antennas and $\mathcal{Q}_{I}=\{20,30\} \mathrm{dB}$. The outage probability of the secondary network performance is shown at which the complete co-channel interference imposed by PUs transmitters is achieved. The result for full size arbitrary cancellation and no cancellation can be deduced as limiting cases when $\gamma_{\mathrm{I}, T}=\infty$ and $\gamma_{\mathrm{I}, T}=0$, respectively. It can be observed that when $\gamma_{\mathrm{I}, T}$ increases more interference can be cancelled and hence the system performance is enhanced.

\section{CONCLUSION}

This paper has provided a comprehensive analysis of the impact of interference imposed by mutliple PUs on congitive underlay networks with multihop AF relaying and considering various MIMO techniques per hop. In the adopted secondary network, we have proposed two different transmission schemes including TAS/MRC and TAS/IC (i.e, TAS/DRIC, TAS/ARIC) in order to provide diversity, and to cancel the strongest interference powers, respectively. In doing so, we have derived the exact and asymptotic outage expressions, which provide an efficient means to evaluate the impact of the number of PU transmitter and receivers, antenna array sizes at SU network, the number of hops, interference outage constrains, 


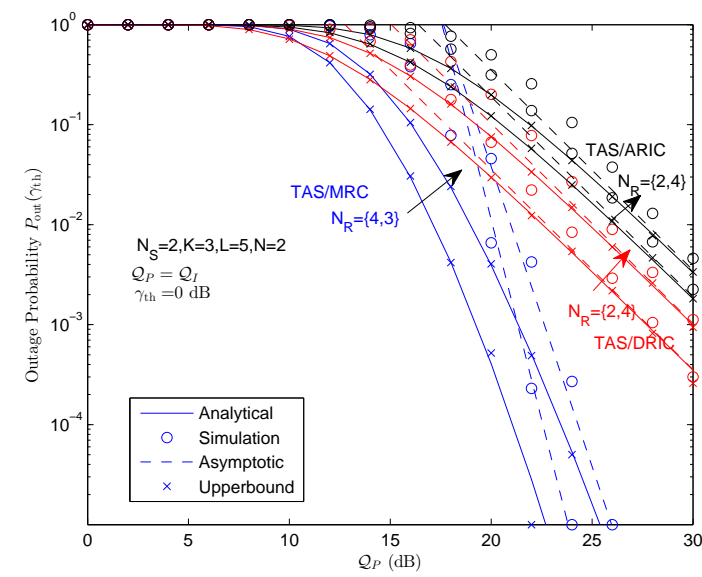

Fig. 3. The outage probability of cognitive MIMO multihop in spectrum sharing with TAS/MRC, TAS/DRIC, and TAS/ARIC when $\mathcal{Q}_{P}=\mathcal{Q}_{I}$.

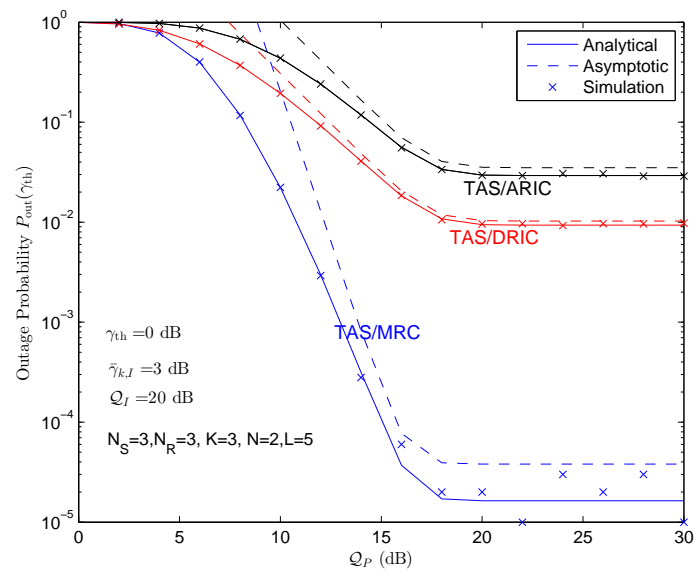

Fig. 4. The outage probability of cognitive MIMO multihop in spectrum sharing with TAS/MRC, TAS/DRIC, and TAS/ARIC when $\mathcal{Q}_{I}$ is fixed.

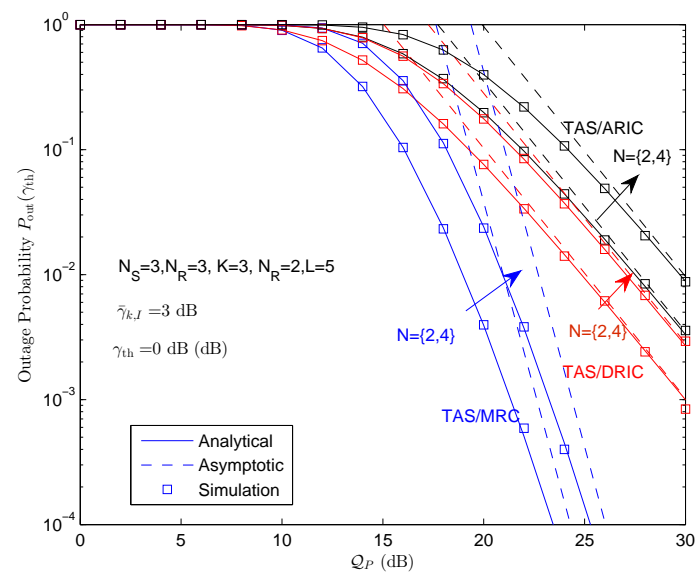

Fig. 5. The outage probability of cognitive MIMO multihop in spectrum sharing with TAS/MRC, TAS/DRIC, and TAS/ARIC when $\mathcal{Q}_{P}=\mathcal{Q}_{I}$ and $N$ is varied.

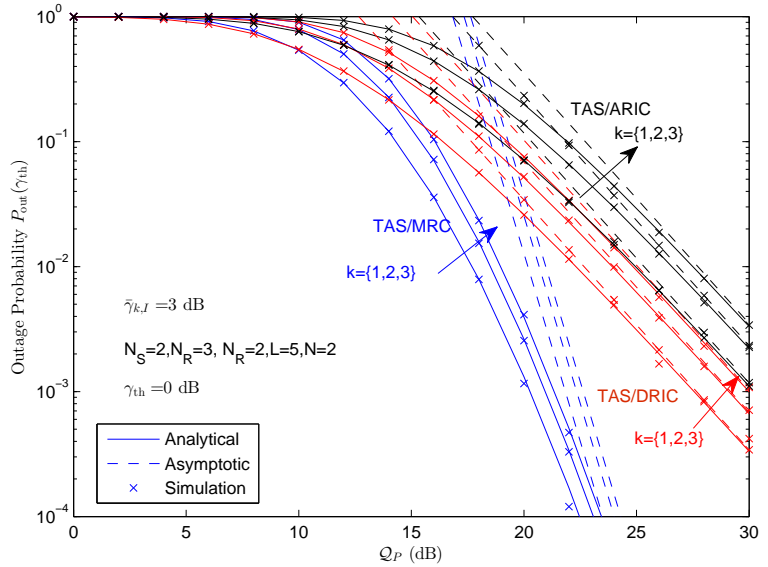

Fig. 6. The outage probability of cognitive MIMO multihop in spectrum sharing with TAS/MRC, TAS/DRIC, and TAS/ARIC when $\mathcal{Q}_{P}=\mathcal{Q}_{I}$ and number of hops is varied.

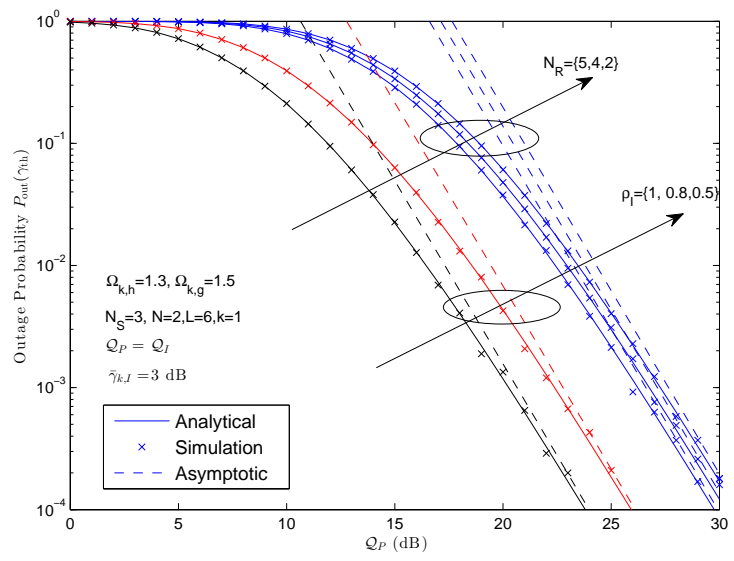

Fig. 7. The outage probability of cognitive MIMO multihop in spectrum sharing with dominant interference reduction when $\mathcal{Q}_{P}=\mathcal{Q}_{I}$, and $\bar{\gamma}_{k, \mathrm{I}}=$ $3 \mathrm{~dB}$.

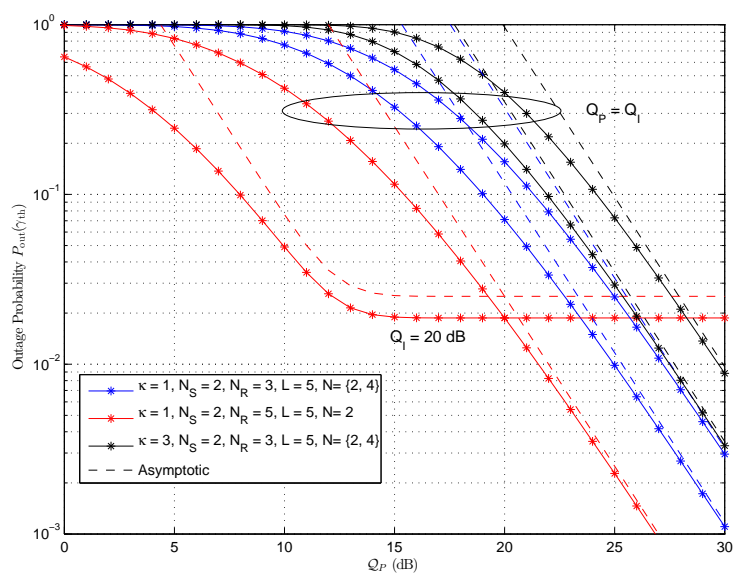

Fig. 8. The outage probability of cognitive MIMO multihop in spectrum sharing with ARIC when $\bar{\gamma}_{k, T}=3 \mathrm{~dB}, \mathcal{Q}_{I}$ is fixed and varied $\mathcal{Q}_{I}=\mathcal{Q}_{P}$. 


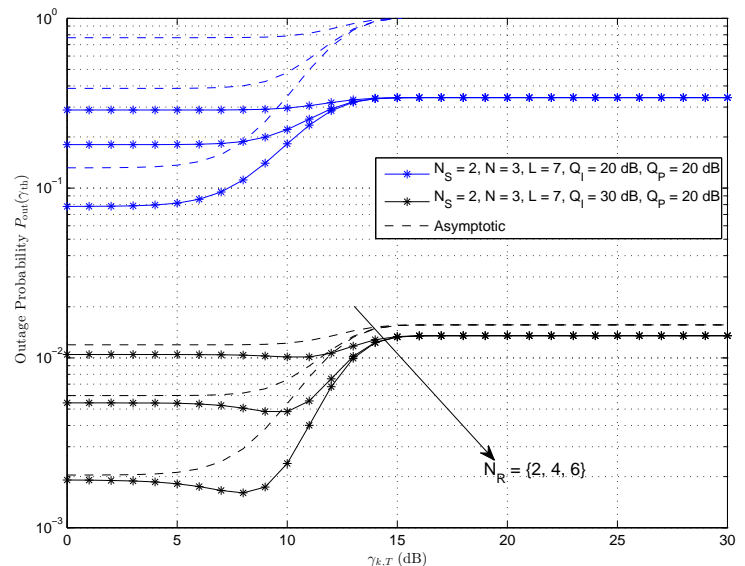

Fig. 9. The outage probability of cognitive MIMO multihop in spectrum sharing with ARIC when $\mathcal{Q}_{I}$ is fixed.

and interference cancellation schemes on the outage performance of the considered systems. Based on the new results, important insights are revealed into the relation between the maximum SU transmit power $\mathcal{Q}_{P}$, and the peak interference temperature $\mathcal{Q}_{I}$. When $\mathcal{Q}_{I}$ is proportional to $\mathcal{Q}_{P}$, TAS/MRC achieves a diversity of $N_{S} N_{R}$ gain, and both TAS/IC cancellation schemes attain $N_{S}$ gain which as a result shows that TAS/MRC outperforms TAS/IC schemes in the high SNR. While TAS/DRIC achieves better performance in the low SNR. However, both diversity and cancellation schemes perform almost the same in the low SNR (i.e., $<10 \mathrm{~dB}$ ). When $\mathcal{Q}_{I}$ is fixed, the outage floor appears, and hence zero diversity gains for both TAS/MRC and TAS/IC scenarios.

\section{APPENDIX}

\section{A. Proof of Theorem 1}

The CDF of $\gamma_{k}$ can be obtained as [11]

$$
\begin{aligned}
F_{\gamma_{k}}\left(\gamma_{\mathrm{th}}\right) & =\underbrace{\operatorname{Pr}\left\{\frac{\mathcal{Q}_{P} \lambda_{k, h}}{\gamma_{k, \mathrm{I}}+1} \leq \gamma_{\mathrm{th}}, \mathcal{Q}_{P} \leq \frac{\mathcal{Q}_{I}}{\lambda_{k, g}}\right\}}_{\mathcal{I}_{1}} \\
& +\underbrace{\operatorname{Pr}\left\{\frac{\mathcal{Q}_{I} \lambda_{k, h}}{\lambda_{k, g}\left(\gamma_{k, \mathrm{I}}+1\right)} \leq \gamma_{\mathrm{th}}, \frac{\mathcal{Q}_{I}}{\lambda_{k, g}} \leq \mathcal{Q}_{P}\right\}}_{\mathcal{I}_{2}} .
\end{aligned}
$$

The first summand can be evaluated as

$$
\mathcal{I}_{1}=F_{\lambda_{k, g}}\left(\frac{\mathcal{Q}_{I}}{\mathcal{Q}_{P}}\right) \int_{0}^{\infty} F_{\lambda_{k, h}}\left(\frac{\gamma_{\mathrm{th}}(z+1)}{\mathcal{Q}_{P}}\right) f_{\gamma_{k, \mathrm{I}}}(z) d z
$$

With the appropriate substitution of the distributions of $\lambda_{k, g}$ and $\gamma_{k, \mathrm{I}}$ into (46), and with the help of [33, 3.351.3], the term $\mathcal{I}_{1}$ can be evaluated in closed-form expression as

$$
\begin{aligned}
& \mathcal{I}_{1}=F_{\lambda_{k, g}}\left(\frac{\mathcal{Q}_{I}}{\mathcal{Q}_{P}}\right) \\
& \left(1-\frac{\left(\frac{1}{\bar{\gamma}_{k, \mathrm{I}}}\right)^{L}}{\Gamma(L)} \sum_{k_{1}=1}^{N_{S}}\left(\begin{array}{c}
N_{S} \\
k_{1}
\end{array}\right)(-1)^{\left(k_{1}-1\right)} \Gamma\left(k_{1}+1\right) e^{-\gamma_{\mathrm{th}}\left(\frac{k_{1}}{\Omega_{k, h} Q_{P}}\right)}\right. \\
& \left.\sum_{\left|\mathbf{c}_{\mathbf{p}}\right|=k_{1}} \mu_{p}\left(\frac{\gamma_{\mathrm{th}}}{\Omega_{k, h} Q_{P}}\right)^{\lambda_{p}} \sum_{v=0}^{\lambda_{p}}\left(\begin{array}{c}
\lambda_{p} \\
v
\end{array}\right) \frac{\Gamma(L+v)}{\left(\frac{k_{1} \gamma_{\mathrm{th}}}{\Omega_{k, h} Q_{P}}+\frac{1}{\bar{\gamma}_{k, \mathrm{I}}}\right)^{(L+v)}}\right) .
\end{aligned}
$$

For $\mathcal{I}_{2}$, it gives

$$
\mathcal{I}_{2}=\int_{0}^{\infty}(\underbrace{\int_{\frac{\mathcal{Q}_{I}}{\mathcal{Q}_{P}}}^{\infty} F_{\lambda_{k, h}}\left(\frac{\gamma_{\mathrm{th}} y(z+1)}{\mathcal{Q}_{I}}\right) f_{\lambda_{l, g}}(y) d y}_{\mathcal{I}_{3}}) f_{\gamma_{k, \mathrm{I}}}(z) d z
$$

Starting with the inner integral $\mathcal{I}_{3}$, by performing appropriate substitution of $\lambda_{k, g}$ into (48), and averaging over the distribution of $\lambda_{k, g}$, it gives (49) as shown on the top of the next page, where the integral can be obtained in closedform expression with the help of $[33,3.351 .3]$ as shown in (49). Then by inserting (50) into $\mathcal{I}_{2}$, performing the averaging over the distribution $\gamma_{k, \mathrm{I}}$, and then after careful mathematical manipulations with the use of the identity in [33, 3.471.7], the final result for $\mathcal{I}_{2}$ can be obtained.

\section{B. Proof of Proposition 1}

By utilizing the Maclaurin series expansion of the exponential function and neglecting the higher order function, the CDF of $\lambda_{k, h}$ can be approximated by

$$
F_{\lambda_{k, h}}(x) \approx\left(\frac{\left(\frac{x}{\Omega_{k, h}}\right)^{N_{R}}}{\Gamma\left(N_{R}+1\right)}\right)^{N_{S}}
$$

By inserting the approximated CDF of $\lambda_{k, h}$ in (51) into (46) and (48), then the terms $\mathcal{I}_{1}$ and $\mathcal{I}_{2}$ can be given respectively by

$\mathcal{I}_{1}=F_{\lambda_{k, g}}\left(\frac{\mathcal{Q}_{I}}{\mathcal{Q}_{P}}\right) \frac{\left(\frac{\gamma_{\mathrm{th}}}{\mathcal{Q}_{P} \Omega_{k, h}}\right)^{N_{S}}}{\Gamma\left(N_{R}+1\right)^{N_{S}}} \int_{0}^{\infty}(z+1)^{N_{S}} f_{\gamma_{k, \mathrm{I}, \mathrm{tot}}}(z) d z$

and

$$
\begin{aligned}
\mathcal{I}_{2} & =\frac{\left(\frac{\gamma_{\mathrm{th}}}{\mathcal{Q}_{I} \Omega_{k, h}}\right)^{N_{S}}}{\Gamma\left(N_{R}+1\right)^{N_{S}}}\left(\int_{0}^{\infty}(z+1)^{N_{S}} f_{\gamma_{k, \mathrm{I}}}(z) d z\right) \\
& \times\left(\int_{\frac{\mathcal{Q}_{I}}{\mathcal{Q}_{P}}}^{\infty} y^{N_{S}} f_{\lambda_{k, g}}(y) d y\right)
\end{aligned}
$$

By performing some mathematical manipulations, and with the help of [33, 3.351.3] and [33, 3.471.7], the final asymptotic expression in Proposition in (1) is obtained. 


$$
\begin{aligned}
& \mathcal{I}_{3}=1-\frac{L}{\Gamma(N) \Omega_{k, g}} \sum_{k_{1}=1}^{N_{S}}\left(\begin{array}{c}
N_{S} \\
k_{1}
\end{array}\right)(-1)^{k_{1}-1} \Gamma\left(k_{1}+1\right) \sum_{\left|\mathbf{c}_{\mathbf{p}}\right|=k_{1}} \mu_{p} \sum_{k_{3}=0}^{L-1}\left(\begin{array}{c}
L-1 \\
k_{3}
\end{array}\right)(-1)^{k_{3}} \Gamma\left(k_{3}+1\right) \\
& \times \sum_{\left|\mathbf{c}_{\mathbf{1}}\right|=k_{3}} \mu_{l}\left(\frac{1}{\Omega_{k, g}}\right)^{\lambda_{l}+N-1}\left(\frac{\gamma_{\mathrm{th}}(1+z)}{\Omega_{k, h} Q_{I}}\right)^{\lambda_{p}} \int_{\frac{Q_{I}}{Q_{P}}}^{\infty} y^{\lambda_{p}+\lambda_{l}+N-1} \exp \left(-y\left(\frac{\gamma_{\mathrm{th}}\left(1+\gamma_{I}\right) k_{1}}{\Omega_{k, h} Q_{I}}+\frac{\left(k_{3}+1\right)}{\Omega_{k, g}}\right)\right) d y \\
& =1-\frac{L}{\Gamma(N) \Omega_{k, g}} \sum_{k_{1}=1}^{N_{S}}\left(\begin{array}{c}
N_{S} \\
k_{1}
\end{array}\right)(-1)^{k_{1}-1} \Gamma\left(k_{1}+1\right) \sum_{\left|\mathbf{c}_{\mathbf{p}}\right|=k_{1}} \mu_{p} \sum_{k_{3}=0}^{L-1}\left(\begin{array}{c}
L-1 \\
k_{3}
\end{array}\right)(-1)^{k_{3}} \Gamma\left(k_{3}+1\right) \\
& \times \sum_{\left|\mathbf{c}_{\mathbf{l}}\right|=k_{3}} \mu_{l}\left(\frac{1}{\Omega_{k, g}}\right)^{\lambda_{l}+N-1} \theta^{\lambda_{p}} e^{-\frac{Q_{I}}{Q_{P}} \phi} \sum_{k=0}^{\mu-1} \frac{\Gamma(\mu)}{\Gamma(k+1)}\left(\frac{Q_{I}}{Q_{P}}\right)^{k}\left(\frac{1}{\phi}\right)^{\mu-k} \sum_{\zeta=0}^{\lambda_{p}}\left(\begin{array}{c}
\lambda_{p} \\
\zeta
\end{array}\right) \gamma_{I}^{\zeta} e^{-\left(\frac{Q_{I} k_{1} \theta}{Q_{P}}\right) z}\left(1+\frac{k_{1} \theta}{\phi} z\right)^{-(\mu-k)}
\end{aligned}
$$

\section{Proof of Corollary (2)}

The DMT can be evaluated according to [25]

$$
d(\hat{r})=\lim _{\mathcal{Q}_{P} \rightarrow \infty} \frac{-\log P_{\text {out }}\left(\hat{r}, \mathcal{Q}_{P}\right)}{\log \mathcal{Q}_{P}} .
$$

where $\hat{r}=R / \log \left(1+\mathcal{Q}_{P}\right)$ is the normalized spectral efficiency with respect to the channel capacity, and $R=$ $1 / K \log \left(1+\gamma_{\text {th }}\right)$ is the spectral effiency in bits/s/Hz with pre$\log$ factor due to the $K-1$ number of hops. Then, invoking the asymptotic outage approximation given in Proposition 1 , we have

$$
\begin{aligned}
d(\hat{r}) & =N_{S} N_{R} \\
& \times\left[1-\lim _{\mathcal{Q}_{P} \rightarrow \infty} \frac{\log \left(\left(1+\mathcal{Q}_{P}\right)^{2 \hat{r}}-1\right)}{\log \mathcal{Q}_{P}}\right] .
\end{aligned}
$$

To this end, applying the L'Hospital's rule yields the desired result in Corollary (2).

\section{Proof of Proposition (3)}

Based on the optimization problem in (19), the Lagrange cost furcation can be written as

$$
\begin{aligned}
\mathcal{L}\left(P_{k}, \phi, \beta\right) & =\sum_{k=1}^{K} \Psi_{k}\left(\frac{\gamma_{\mathrm{th}}}{\mathcal{Q}_{P}}\right)^{N_{S} N_{R}}+\phi\left(\sum_{k=1}^{K} P_{k}-P_{\text {tot }}\right) \\
& +\beta\left(\sum_{k=1}^{K} \lambda_{k, g} P_{k}-I_{P}\right)
\end{aligned}
$$

where $\phi$ and $\beta$ are the lagrange parameters. Upon setting the derivatives of with respect to $P_{k}, \phi$ and $\beta$ to zero, we get

$$
\begin{aligned}
& \frac{\mathcal{L}\left(P_{k}, \psi, \phi\right)}{\partial P_{k}}=\frac{-N_{S} N_{R} \Psi_{k}\left(\gamma_{\mathrm{th}}\right)^{N_{S} N_{R}}}{P_{k}^{N_{S} N_{R}+1}}+\phi+\beta \lambda_{k, g}=0 \\
& \text { for } \quad k=1 \ldots K \\
& \frac{\mathcal{L}\left(P_{k}, \psi, \phi\right)}{\partial \phi}=\sum_{k=1}^{K} P_{k}-P_{\text {tot }}=0 \\
& \frac{\mathcal{L}\left(P_{k}, \psi, \phi\right)}{\partial \beta}=\sum_{k=1}^{K} \lambda_{k, g} P_{k}-I_{P}=0
\end{aligned}
$$

Now, by solving the set of $k+2$ equations simultaneously, we reach the final result in Proposition 3.

\section{E. Proof of Theorem 3}

To evaluate the end-to-end SINR $\gamma_{k}$, we need to evaluate $\mathcal{I}_{1}$ and $\mathcal{I}_{2}$ in (46) and (48), respectively. Therefore, the integral term $\mathcal{I}_{1}$ can be expressed as

$$
\begin{aligned}
\mathcal{I}_{1} & =F_{\lambda_{k, g}}\left(\frac{\mathcal{Q}_{I}}{\mathcal{Q}_{P}}\right) \int_{\gamma_{\mathrm{I}, T}}^{\infty} F_{\lambda_{k, h}}\left(\frac{\gamma_{\mathrm{th}}(z+1)}{\mathcal{Q}_{P}}\right) f_{\gamma_{k, \mathrm{I}, \mathrm{tot}}}^{z>\gamma_{\mathrm{I}, T}}(z) d z \\
& +\int_{0}^{\gamma_{\mathrm{I}, T}} F_{\lambda_{k, h}}\left(\frac{\gamma_{\mathrm{th}}(z+1)}{\mathcal{Q}_{P}}\right) f_{\gamma_{k, \mathrm{I}, \mathrm{tot}}}^{z \leq \gamma_{\mathrm{I}, T}}(z) d z
\end{aligned}
$$

where $f_{\gamma_{\mathrm{k}, \mathrm{I}, \mathrm{tot}}}^{z>\gamma_{\mathrm{I}, T}}(z)$ and $f_{\gamma_{\mathrm{k}, \mathrm{I}, \mathrm{tot}}}^{z \leq \gamma_{\mathrm{I}, T}}(z)$ are given respectively by

$$
f_{\gamma_{\mathrm{k}, \mathrm{I}, \mathrm{tot}}}^{z>\gamma_{\mathrm{I}, T}}(z)=\frac{1}{\Gamma\left(L_{A}\right)}\left(\frac{1}{\bar{\gamma}_{k, \mathrm{I}}}\right)^{L_{A}} z^{L_{A}-1} e^{-z / \bar{\gamma}_{k, \mathrm{I}}},
$$

and

$$
\begin{aligned}
f_{\gamma_{\mathrm{k}, \mathrm{I}, \mathrm{tot}}}^{z \leq \gamma_{\mathrm{I}}, T}(z) & =\frac{1}{\Gamma(L)}\left(\frac{1}{\bar{\gamma}_{k, \mathrm{I}}}\right)^{L} z^{L-1} e^{-z / \bar{\gamma}_{k, \mathrm{I}}} \\
& +e^{-\gamma_{\mathrm{I}, T} / \bar{\gamma}_{k, \mathrm{I}}} \sum_{h=2}^{L_{R}} \frac{\left(\frac{1}{\bar{\gamma}_{k, \mathrm{I}}}\right)^{\iota}}{\Gamma(\iota)} z^{\iota-1} .
\end{aligned}
$$

With the appropriate substitution of the distributions of $\lambda_{k, g}$ and $\gamma_{k, \text { I,tot }}$ into (46), and with the help of [33, 3.381.1] and [33, 3.381.2], the first and the second integrals can be evaluated in closed-from expressions, respectively. After evaluating $\mathcal{I}_{1}$, we turn our attention to $\mathcal{I}_{2}$, it gives

$\mathcal{I}_{2}=\int_{0}^{\infty}\left(\int_{\frac{\mathcal{Q}_{I}}{\mathcal{Q}_{P}}}^{\infty} F_{\lambda_{k, h}}\left(\frac{\gamma_{\mathrm{th}} y(z+1)}{\mathcal{Q}_{I}}\right) f_{\lambda_{l, g}}(y) d y\right) f_{\gamma_{k, \mathrm{I}, \mathrm{tot}}}(z) d z$

Starting with the inner integral, by performing appropriate substitution of $\lambda_{k, h}$ into (61), and averaging over the distribution of $\lambda_{k, g}$, the inner integral i.e., $\mathcal{I}_{3}$ integral can be evaluated in 
closed-form with the help of $[33,3.351 .3]$ as

$$
\begin{aligned}
& \mathcal{I}_{3}= \frac{L}{\Gamma(N) \Omega_{k, g}} \sum_{k_{1}=0}^{N_{S}}\left(\begin{array}{c}
N_{S} \\
k_{1}
\end{array}\right)(-1)^{k_{1}} \sum_{k_{3}=0}^{L-1}\left(\begin{array}{c}
L-1 \\
k_{3}
\end{array}\right) \frac{\Gamma\left(k_{3}+1\right)}{(-1)^{-k_{3}}} \\
& \sum_{\left|\mathbf{c}_{1}\right|=k_{3}} \mu_{l}\left(\frac{1}{\Omega_{k, g}}\right)^{\lambda_{l}+N-1} \exp \left(-\frac{Q_{I}}{Q_{P}} \phi\right) \sum_{k=0}^{\mu_{2}-1} \frac{\Gamma\left(\mu_{2}\right)}{\Gamma(k+1)} \\
& \quad \times\left(\frac{Q_{I}}{Q_{P}}\right)^{k}\left(\frac{1}{\phi}\right)^{\mu_{2}-k} \frac{\exp \left(-\frac{Q_{I} k_{1} \theta}{Q_{P}} z\right)}{\left(1+\frac{k_{1} \theta}{\phi} z\right)^{\left(\mu_{2}-k\right)}}
\end{aligned}
$$

Then the outer integral can be solved by averaging over the distribution $\gamma_{k, \mathrm{I} \text {,tot }}$ as

$$
\begin{aligned}
\mathcal{I}_{2}= & \frac{L}{\Gamma(N) \Omega_{k, g}} \sum_{k_{1}=0}^{N_{S}}\left(\begin{array}{c}
N_{S} \\
k_{1}
\end{array}\right)(-1)^{k_{1}} \sum_{k_{3}=0}^{L-1}\left(\begin{array}{c}
L-1 \\
k_{3}
\end{array}\right) \frac{\Gamma\left(k_{3}+1\right)}{(-1)^{-k_{3}}} \\
& \sum_{\left|\mathbf{c}_{\mathbf{l}}\right|=k_{3}} \mu_{l}\left(\frac{1}{\Omega_{k, g}}\right)^{\lambda_{l}+N-1} \exp \left(-\frac{Q_{I}}{Q_{P}} \phi\right) \sum_{k=0}^{\mu_{2}-1} \frac{\Gamma\left(\mu_{2}\right)}{\Gamma(k+1)} \\
& \times\left(\frac{Q_{I}}{Q_{P}}\right)^{k}\left(\frac{1}{\phi}\right)^{\mu_{2}-k}\left(\int_{0}^{\gamma_{\mathrm{I}, T}} \frac{\exp \left(-\frac{Q_{I} k_{1} \theta}{Q_{P}} z\right)}{\left(1+\frac{k_{1} \theta}{\phi} z\right)^{\left(\mu_{2}-k\right)} f_{\gamma_{k, \mathrm{I}, \mathrm{tot}}}^{z \leq \gamma_{\mathrm{I}, T}}(z)}\right. \\
& +\int_{\gamma_{\mathrm{I}, T}}^{\infty} \frac{\exp \left(-\frac{Q_{I} k_{1} \theta}{Q_{P}} z\right)}{\left(1+\frac{k_{1} \theta}{\phi} z\right)^{\left(\mu_{2}-k\right)} f_{\gamma_{\mathrm{k}, \mathrm{I}, \mathrm{tot}} \leq \gamma_{\mathrm{I}, T}(z)}}
\end{aligned}
$$

To evaluate the above integrals, we perform change of variable $u=\left(1+\frac{k_{1} \theta}{\phi} z\right)$ and applying formula [33, 3.471.7], the final solution of $\mathcal{I}_{2}$ is obtained. To this end, after evaluating $\mathcal{I}_{1}$ and $\mathcal{I}_{2}$, the desired results for Theorem (3) derived.

\section{REFERENCES}

[1] S. S. Ikki and S. Aissa, "Multihop wireless relaying systems in the presence of co-channel interference: performance analysis and design optimization,"IEEE Trans. Veh. Technol., vol. 61, no. 2, pp. 566-573, Feb. 2012.

[2] A. M Salhab, F. S. Al-Qahtani, S. A Zummo, H. Alnuweiri, ”Exact Outage Probability of Opportunistic DF Relay Systems With Interference at Both the Relay and the Destination Over Nakagami-Fading Channels,"IEEE Trans. Veh. Technol., vol. 61, no. 2, pp. 920-927, Feb. 2013.

[3] I. F. Akyildiz, W.-Y. Lee, M. C. Vuran, and S. Mohanty, "Next generation dynamic spectrum access cognitive radio wileless networks: a survey," Comput. Netw., vol. 50, no. 13, pp. 2127-2159, Sep. 2006.

[4] Q. Zhao and B. M. Sadler, "A survey of dynamic spectrum access," IEEE Signal Process. Mag., vol. 24, no. 3, pp. 79-89, 2007.

[5] A. Ghasemi and E. S. Sousa, "Fundamental limits of spectrum-sharing in fading environments," IEEE Trans. Wireless Commun., vol. 9, no. 2, pp. $649-658$, Feb. 2007.

[6] G. V. Tsoulos, Adaptive Antennas for Wireless Communications, WileyIEEE Press, 2001

[7] J. Lee, H. Wang, J. G. Andrews, and D. Hong, "Outage probability of cognitive relay networks with interference constraints," IEEE Trans. Wireless Commun., vol. 10, no. 2, pp. 390-395, Feb. 2011.

[8] C. Zhong, T. Ratnarajah, and K.-K. Wong, "Outage analysis of decodeand-forward cognitive dual-hop systems with the interference constraint in Nakagami-m fading channels," IEEE Trans. Veh. Technol., vol. 60, no. 6, pp. 2875-2879, July 2011.

[9] Wei. Xu, J. Zhang, P. Zhang, and C. Tellambura, "Outage probability of decode-and-forward cognitive relay in presence of primary user's interference," IEEE Commun. Lett., vol. 16, no. 8, pp. 1252-1255, Aug. 2012.
[10] T. Q. Duong, D. B. da Costa, T. A. Tsiftsis, C. Zhong, and A. Nallanathan, "Outage and diversity of cognitive relaying systems under spectrum sharing environments in Nakagami- $m$ fading," IEEE Commun. Lett., vol. 16, no. 12, pp. 2075-2078, Dec. 2012.

[11] T. Q. Duong, V. N. Q. Bao, and H.-J. Zepernicj, "Exact outage probability of cognitive AF relaying with undelay spectrum sharing," Electronic letters, vol. 47, no. 17, pp. 1001-1002, Jul. 2011.

[12] T. Q. Duong, P. Lep Yeoh, V. N. Q. Bao, M. Elkashlan, and N. Yang, "Cognitive Relay Networks With Multiple Primary Transceivers Under Spectrum-Sharing," IEEE Signal process. Lett., vol. 19, no. 11, Nov. 2012.

[13] Kim, K. J, T. Q. Duong, T. A. Tsiftsis, V. N. Q. Bao, "Cognitive multihop networks in spectrum sharing environment with multiple licesed users," in Proc. IEEE ICC, 2013.

[14] A. Hyadi, M. Benjillali, M.-S. Alouini, and D. da Costa, "Performance analysis of underlay cognitive multihop regenerative relaying systems with multiple primary receivers," IEEE Trans. Commun., vol. 12, no. 12, pp. 64186429, Dec. 2013.

[15] P. Bithas, and A. Rontogiannis, "outage probability analysis of multi hop cognitive networks under multiple primary user interference," in Proc. European Wireless, 2015.

[16] P. L. Yeoh, M. Elkashlan, T. Q. Duong, N. Yan, and D. B. da Costa, "Transmit antenna selection in cognitive relay networks with Nakagami$m$ fading," in Proc. IEEE ICC, 2013.

[17] P. L. Yeoh, M. Elkashlan, T. Q. Duong, N. Yan, and D. B. da Costa, "Transmit antenna selection for interference management in cognitive relay networks," in IEEE Trans. Veh. Technol., vol. 63, no. 7, Sept. 2013.

d현 F. S Al-Qahtani, Y. Huang, M. Di Renzo, S. S. Ikki, H. Alnuweiri, "Space shift keying MIMO system under spectrum sharing environments in Rayleigh fading," in IEEE Commun. Lett., vol. 18, no. 9, pp. 15031506, Sept. 2014.

[19] Y. Huang, F. S Al-Qahtani, Q. Wu, C. Zhong, J. Wang, H. Alnuweiri, "Outage Analysis of Spectrum Sharing Relay Systems With Multiple Secondary Destinations Under Primary User's Interference," in IEEE Trans. Veh. Technol., vol. 63, no. 7, pp. 3456-3464, Sept. 2014.

[20] R. M. Radaydeh, "Performance analysis of rectangular quadrature amplitude modulation with combined arbitrary transmit antenna selection and receive maximal ratio combining in nakagami-m fading," IET Commun. vol. 2, no. 8, pp. 1077-1088, Sept. 2008.

[21] R. M. Radaydeh, "Receive MRC with Outdated Arbitrary Transmit Antenna Selection in Nakagami-m Fading," IET Communications, vol. 3 , no. 10 , pp. $1638-1648$, Oct. 2009.

[22] R. M. Radaydeh, "Impact of Delayed Arbitrary Transmit Antenna Selection on the Performance of Rectangular QAM with Receive MRC in Fading Channels," IEEE Commun. Lett., vol. 13, no. 6, pp. 390-392, Jun. 2009.

[23] B. Allen and M. Ghavami, Adaptive Array Systems: Fundamentals and Applications, John Wiley \& Sons Ltd, 2005.

[24] J. A. Ritcey and M. Azizoglu, "Performance analysis of generalized selection combining with switching constraints," IEEE Commun. Lett., vol. 4, pp. 152-154, May 2000.

[25] J. N. Laneman, D. N. C. Tse, and G. W. Wornell, "Cooperative diversity in wireless networks: Efficient protocols and outage behavior," IEEE Trans. Inf. Theory, vol. 50, no. 12, pp. 3062-3080, Dec. 2004.

[26] N. I. Miridakis and D. D. Vergados, "A Survey on the Successive Interference Cancellation Performance for Single-Antenna and MultipleAntenna OFDM Systems" IEEE Communications Surveys \& Tutorials, vol. 15 , No. 1,2013

[27] M. O. Hasna, M.-S. Alouini, A. Bastami, and E. S Ebbini, "Performance analysis of cellular mobile radio systems with succesive co-channel interference cancellation," IEEE Trans. Wireless Commun., vol. 2, no. 1, pp. 29-49, Jan. 2003.

[28] R. M. Radaydeh and M.-S. Alouini, "Adaptive co-channel interference cancelation for power-limited applications," in Proc. IEEE Int. Symp. on Personal, Indoor and Mobile Radio Commun. (PIRMC'2010), Istanbul, Turkey, Sept. 2010.

[29] R. M. Radaydeh and M.-S. Alouini,, "Performance Analysis of PowerEfficient Adaptive Interference Cancelation in Fading Channels," in Proc. IEEE Global Telecomm. Conf., 2010, pp. 1-5.

[30] R. M. Radaydeh and M.-S. Alouini, "On the Performance of Arbitrary Transmit Selection for Threshold-Based Receive MRC with and without Co-Channel Interference," IEEE Trans. Commun., vol. 59, no. 11, pp. 3177-3191, 2011

[31] R. M. Radaydeh and M.-S. Alouini, "Comparisons of Receive Array Interference Reduction Techniques Under Erroneous Generalized Transmit 
Beamforming," IEEE Trans. Commun., vol. 62, no. 1, pp. 600-615, Feb. 2014.

[32] S. Choi and Y.-C. Ko, "Performance of selection MIMO systems with generalized selection criterion over Nakagamim fading channels," IEICE Trans. Commun., vol. E89-B, pp. 3467-3470, Dec. 2006.

[33] I. S. Gradshteyn and I. M. Ryzhik, Table of Integrals, Series and Products, 6th ed. San Diego, CA: Academic, 2000.

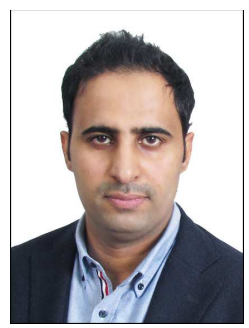

Fawaz S. Al-Qahtani (M10) received the B.Sc. degree in electrical engineering from King Fahad University of Petroleum and Minerals, Dhahran, Saudi Arabia, the M.Sc. degree in digital communication systems from Monash University, Vic., Melbourne, Australia, and the Ph.D. degree in electrical and computer engineering from RMIT University, Melbourne, Vic., Australia, in 2000, 2005, and 2009, respectively. He is currently an Associate Research Scientist with the Department of Electrical and Computer Engineering, Texas A\&M University at Qatar, Doha, Qatar. His research interests include channel modeling, applied signal processing, MIMO communication systems, cooperative communications, cognitive radio systems, free-space optical, physical-layer security, visible light communication, device-to-device communication, and power transfer. $\mathrm{He}$ is the author and coauthor of over 70 papers in refereed mainstream journals and reputed international conferences. He was the recipient of the sponsorship from the Qatar National Research Fund as well as from JSERP and NPRP projects, and the Research Excellence Award from Texas A\&M University at Qatar, in 2013.

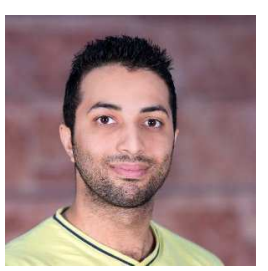

Salah Hessein was born in Cairo, Egypt, in 1988. He received his B.Sc. degree in electrical engineering from Ain Shams University, Cairo, Egypt, in 2010. Salah joined Texas A\&M University in 2013 as a research associate engineer. His current research interests include physical layer security, performance analysis, Monte Carlo simulation of cognitive radio network with particular focus on spectrum sharing techniques and interference mitigation.

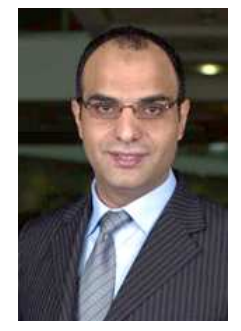

Redha M. Radaydeh (S'05, M'07, SM'13) was born in Irbid, Jordan, on November 12, 1978. He received his B.Sc. (June 2001) and M.Sc. (August 2003) degrees from JUST, and a Ph.D. degree from the University of Mississippi in November 2006, all in Electrical Engineering. His professional career after $\mathrm{PhD}$ includes many academic and research positions. Specifically, from Feb. 2007 to Sept. 2009, he served as an Assistant Professor of Electrical Engineering at JUST. From Sept. 2009 to Sept. 2012, he was a Research Fellow and graduate research supervisor at KAUST, and then he was an Associate Research Scientist at Texas A\&M University in Qatar. From Sept. 2012 to Aug. 2016, he has been with Alfaisal University as an Associate Professor of Electrical Engineering. Currently, he is with KAUST as a research consultant. He has an extensive teaching experience in Electrical Engineering, and contributed widely to the enhancement of study plans, and course specifications, and proposed and taught many advanced courses. His research interests include various research topics on wireless communications and networks, such as MIMO systems, cooperative communications, beamforming and diversity schemes, cognitive radio, small-cell/heterogeneous networks, D2D communication, resource allocation, power adaptation, interference management, information secrecy, and free-space optical and visible light communications. He has authored/coauthored many papers, mainly in leading IEEE Journals and conferences. He has received research funds from many organizations, and received a faculty award for research excellence from Alfaisal University in 2015.

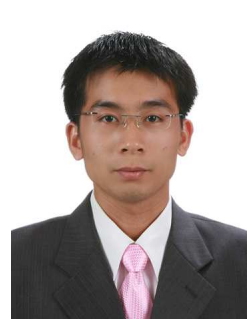

Trung Q. Duong (S'05, M'12, SM'13) received his $\mathrm{Ph} . \mathrm{D}$. degree in Telecommunications Systems from Blekinge Institute of Technology (BTH), Sweden in 2012. Since 2013, he has joined Queen's University Belfast, UK as a Lecturer (Assistant Professor). His current research interests include small-cell networks, physical layer security, energy-harvesting communications, cognitive relay networks. He is the author or co-author of more than 220 technical papers published in scientific journals (110 articles) and presented at international conferences $(110 \mathrm{pa}-$ pers).

Dr. Duong currently serves as an Editor for the IEEE TRANSACTIONS ON Wireless COMmUNications, IEEE TRANSACTIONS ON COMMUNiCATIONS, IEEE COMMUNICATIONS LETTERS, and IET COMMUNICATIONS. He was awarded the Best Paper Award at the IEEE Vehicular Technology Conference (VTC-Spring) in 2013, IEEE International Conference on Communications (ICC) 2014. He is the recipient of prestigious Royal Academy of Engineering Research Fellowship (2016-2021).

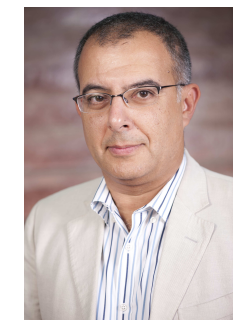

Hussein M. Alnuweiri received the master's degree from King Fahd University of Petroleum and Minerals, Dhahran, Saudi Arabia, in 1984, and the Ph.D. degree in electrical and computer engineering from the University of Southern California, Los Angeles, in 1989.

He is currently a Professor and Program Chair of the Department of Electrical and Computer Engineering, Texas A\&M University, Doha, Qatar. From 1991 to 2007, he was a Professor with the Department of Electrical and Computer Engineering, University of British Columbia. From 1996 to 1998, he also represented the University of British Columbia, Vancouver, BC, Canada, at the ATM Forum. From 2000 to 2006, he served as a Canadian delegate to the ISO/IEC JTC1/SC29 Standards Committee (MPEG-4 Multimedia Delivery), where he worked within the MPEG-4 standardization JTC1-SC29WG11 and the AdHoc group involved in the development of the reference software IM1 AHG.

Dr. Alnuweiri has a long record of industrial collaborations with several major companies worldwide. He is also an inventor, and holds three U.S. patents and one international patent. He has authored or co-authored over 150 refereed journal and conference papers in various areas of computer and communications research. In particular, his research interests include mobile Internet technologies, multimedia communications, wireless protocols, routing and information dissemination algorithms for opportunistic networking, and quality-of-service provisioning and resource allocation in wireless networks. 